\title{
High-Reynolds-number asymptotics of the steady flow through a row of bluff bodies
}

\author{
By S. I. CHERNYSHENKO ${ }^{1}$ AND IAN P. CASTRO \\ ${ }^{1}$ Institute of Mechanics, Moscow University, 117192 Moscow, Russia \\ ${ }^{2}$ Department of Mechanical Engineering, University of Surrey, Guildford GU2 5XH, UK
}

(Received 3 January 1992 and in revised form 22 April 1993)

An extension of an earlier theory of the two-dimensional incompressible flow past an isolated body is described. For a crossflow cascade of bodies, each of unit size in the crossflow direction and distance $2 H$ apart, the region of validity of the extended theory covers $H \gg 1$. A comparison with recent numerical calculations is favourable and a tentative asymptotic structure for the case of $H=O(1)$ is described.

\section{Introduction}

Steady flows around a cascade of bluff bodies have recently attracted considerable attention. For example, theoretical investigations were made by Smith (1985a) and Milos \& Acrivos (1986) and numerical calculations have been reported by Milos, Acrivos \& Kim (1987), Ingham, Tang \& Morton (1990), Fornberg (1991) and Natarajan, Fornberg \& Acrivos (1992). The latest overview of the subject is given by Fornberg (1993). More references can be found in these papers.

The problem under consideration is that of the asymptotic behaviour of the twodimensional steady solution to the Navier-Stokes equations at large Reynolds numbers, $R e$, for the incompressible flow through an infinite row of bluff bodies located at equal distances across the flow. All bodies are supposed to be of the same shape and size and to possess a symmetry axis parallel to the undisturbed flow direction. The theory described in this paper is an extension of the theory of the flow past an isolated body by Chernyshenko (1988). A detailed description of this extended theory is given in the subsequent sections, but it is worth outlining first the main features of the asymptotic structure for the flow past an isolated body.

According to the earlier theory, the eddy behind an isolated body grows indefinitely with $R e$ increasing and the eddy length and width are of the same order of magnitude. On the eddy scale the body shrinks to a point and the flow tends to the well-known Sadovskii solution (with zero jump in the Bernoulli constant across the eddy boundary), which is an inviscid flow past two touching, symmetrical, closed-streamline regions of constant vorticity (by the well-known Prandtl-Batchelor theorem) of equal values and opposite signs. Outside these regions the flow is potential. All such flows are similar and differ in length and velocity scales only (Sadovskii 1970).

Throughout this paper all quantities are non-dimensionalized with the velocity at upstream infinity, the density of the fluid and the characteristic size of the body. Accordingly, the non-dimensional velocity at infinity equals 1 , and the Sadovskii flow is uniquely determined by the eddy length $L$. The vorticity in the eddy $\omega_{\infty}$ is related to $L$ by the formula

$$
\omega_{\infty} L=C_{1} \text {, }
$$

where the constant $C_{1}$ can be found from the Sadovskii (1970) results. 
Another important relation can be obtained using the Bobylev-Forsythe theorem (Serrin 1959), according to which the rate of energy dissipation is proportional to the integral of the vorticity squared, taken over the entire flow field. In the Sadovskii flow the vorticity is non-zero only inside the eddy. Hence, the rate of energy dissipation is proportional to $\omega_{\infty}^{2} L^{2}$. The rate of energy dissipation equals the product of the drag and velocity at infinity. Provided the contribution to the integral from the smaller regions (i.e. the wake) is negligible (this can be proved when the complete structure is known), we eventually arrive at the formula for the drag coefficient

The constant

$$
c_{d}=\text { const } \times \omega_{\infty}^{2} L^{2} / R e=\text { const } \times C_{1}^{2} / R e=C / R e .
$$

$$
C=\int_{-\infty}^{+\infty} \int_{-\infty}^{+\infty} \omega^{2} \partial x \partial y
$$

where $\omega$ is the vorticity in the Sadovskii flow, can be found from the numerical calculation of this flow. Using the well-known expression for the drag in terms of the far-wake characteristics, this formula can also be obtained by considering the wake and the boundary-layer surrounding the eddy, as in the next section.

The third important relation follows from the vorticity balance. The action of viscosity leads to diffusion of vorticity from the eddy. The vorticity is then convected downstream in the wake and it also diffuses towards the symmetry line where it equals zero. This loss of vorticity is compensated by the continuous discharge of vorticity from the body. Naturally, owing to symmetry, the total vorticity flux from the upper and lower parts of the body is zero. Here only one half of the flow (either above or below the symmetry line) is considered. The vorticity flux from the body can be estimated as follows. The Reynolds number based on the characteristic size of the eddy is of order $\operatorname{Re} L$. Accordingly, the thickness of the wake and boundary layer surrounding the eddy is of order $L /(\operatorname{Re} L)^{\frac{1}{2}}=(L / R e)^{\frac{1}{2}}$. As the jump in the Bernoulli constant across the eddy boundary is zero (see later), the vorticity in the recirculating boundary layer has the same order as that in the eddy. Hence, the flux of the vorticity convected downstream to the wake is of order $\omega_{\infty}(L / R e)^{\frac{1}{2}}$. It is easy to verify that the vorticity flux to the symmetry line has the same order. Therefore, vorticity conservation requires the vorticity flux, $F$, from the body to be

$$
F=C_{3} \omega_{\infty}(L / R e)^{\frac{1}{2}} \text {. }
$$

Here the constant $C_{3}$ cannot be found from the Sadovskii results alone: a careful examination of the boundary layer surrounding the eddy is necessary.

The last of the most important relations can be found by analysing the body-scale flow. This flow has been found to be a Kirchhoff flow with free streamlines. The vorticity is convected downstream from the body in the mixing layer near the free streamline. Using a boundary-layer approximation, the vorticity flux can be expressed as

$$
\int u \omega \mathrm{d} n=-\int u \frac{\partial u}{\partial n} \mathrm{~d} n=\frac{1}{2}\left(u_{-}^{2}-u_{+}^{2}\right),
$$

where $u_{-}$and $u_{+}$are the velocities at the mixing-layer boundaries, and the integral is taken across the mixing layer. Therefore the vorticity flux from the body equals the jump in the Bernoulli constant across the free streamline (with the sign reversed). Hence, the velocity on the free streamline is $V_{1 \infty}=(-2 F)^{\frac{1}{2}}$. Therefore the drag coefficient is

$$
c_{d}=k_{d} V_{1 \infty}^{2}=-2 k_{d} F
$$


where $k_{d}$ is the drag coefficient in the Kirchhoff flow which has the velocity on the free streamline equal to that at infinity, i.e. unity.

Now, the system of four equations (1.1)-(1.4) with four unknowns $L, \omega_{\infty}, c_{d}$ and $F$ can be easily solved, giving the main characteristics of the flow. In particular, this yields $L \sim \operatorname{Re}, c_{d} \sim 1 / \operatorname{Re}$.

The extension of the theory to the corresponding cascade flow is straightforward. Assuming the distance between the bodies to be of the same order as the eddy length, the same formulae remain valid with only the constants $C_{1}, C$ and $C_{3}$ changed. The only difference is that instead of the Sadovskii flow in an unbounded domain, the Sadovskii flow in a channel (or, equivalently, a cascade of Sadovskii's flows) must be substituted.

In $\$ \S 2.1-2.3$ the corresponding formulae are thoroughly derived with all the constants determined.

\section{The general structure of the flow}

\subsection{The eddy-scale flow}

Let $H$ be half the distance between the centres of the neighbouring bodies. Locate the origin of the Cartesian coordinate system in the centre of one of the bodies with the $x$-axis directed along the main stream and the $y$-axis along the row. The only difference between this flow and the flow past an isolated body is that a symmetry condition must be implemented at $y= \pm H: \partial u / \partial y=0, v=0$, where $u$ is the $x$-component and $v$ is the $y$-component of the fluid velocity. The flow under consideration depends on the two parameters $R e$ and $H$ and the asymptotics for $R e \rightarrow \infty$ depends on the behaviour of $H$ as a function of $R e$. We will specify this behaviour by the condition that for sufficiently large $R e$ the ratio $H / L$, where $L$ is the eddy length, is fixed. The dependence of $L$ on $R e$ being determined, the resulting asymptotics may be considered as asymptotics for the corresponding $H(R e)$.

Let us assume the length and width of the eddy to be asymptotically large and of the same order. This assumption will be justified only at the very end by the selfconsistency of the expansion. Then in the limit $R e \rightarrow \infty$ the body vanishes on the eddy scale. The resulting inviscid flow (for $H / L=\infty$ ) was considered by Sadovskii $(1971 a$ ); for a given $L$ and $H / L=\infty$ there is a one-parameter family of these flows. The same is presumably true also for a finite $H / L$. The jump, $\Delta$, in Bernoulli's constant across the eddy boundary is a convenient choice of the parameter.

It is possible to proceed further by assuming that $\Delta=0$ and considering the selfconsistency of the resulting solution as the validation of this assumption. Nevertheless an independent proof is sufficiently interesting to be discussed here. Assume for the moment that $\Delta \neq 0$. Then owing to the discontinuity of the velocity at the eddy boundary there is a boundary layer surrounding the eddy. This layer propagates along the eddy boundary from point A (figure 1) to point B. Near this latter point the layer divides. The external part of the layer moves downstream, and the part of the layer below the dividing streamline turns in the vicinity of B and moves from $\mathrm{B}$ to $\mathrm{A}$ along the symmetry line. It turns once more near $A$, joins the external flow and propagates again towards $\mathrm{B}$, and so on.

To formulate the governing equations and boundary conditions for such a layer it is necessary to know the change in the velocity profile in the turn regions near points $A$ and B. The velocity of the inviscid Sadovskii flow tends to zero if point A or B is approached from inside the eddy, and to a non-zero value depending on $\Delta$ if the point is approached from outside (Sadovskii 1971a). Accordingly, the velocity in the 


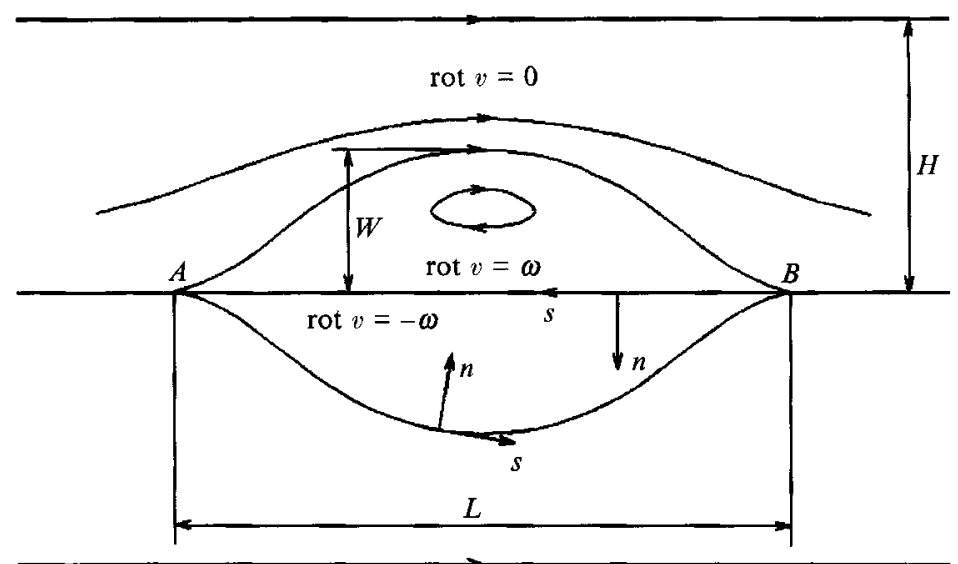

FIGURE 1 . The eddy scale flow with $\Delta \neq 0$. For $\Delta=0$ there are no cusps near points $\mathrm{A}$ and $\mathrm{B}$.

boundary layer approaching these points is $O(1)$, and so is the velocity inside the turn regions. The flow rate through the turn regions is of the same order of magnitude as that in the boundary layer. Hence the lengthscale of the turn regions, which equals the flow rate divided by the velocity scale, has the same order as the boundary-layer thickness. The Reynolds number based on the boundary-layer length, $\delta$, is of order $\operatorname{Re} L$. Therefore $\delta \sim L /(\operatorname{Re} L)^{\frac{1}{2}}$. Hence the Reynolds number based on the lengthscale of the turn region is of order $\operatorname{Re} \delta \sim(\operatorname{Re} L)^{\frac{1}{2}} \rightarrow \infty$. For this reason the flows in the turn regions are effectively inviscid. The size of the turn regions is asymptotically small in comparison with the eddy length, because $\delta / L \sim 1 /(\operatorname{Re} L)^{\frac{1}{2}} \rightarrow 0$.

Hence the pressure at infinity on the turn-region scale equals the pressure in the Sadovskii flow at points A and B. Inside the turn regions the Bernoulli theorem applies owing to the inviscid character of the flow there. Therefore, although the velocity in the turn region changes along a streamline, it returns to the same value at infinity with the pressure returning to its corresponding value. Hence, the dependence of the velocity on the stream function in the boundary layer is the same immediately before and after the turn region. This supplies the boundary conditions for the boundary-layer equations considered below. The derived estimates of the size and Reynolds number of the turn regions are valid for $\Delta \neq 0$, but in $\S 2.4$ similar results concerning the conservation of Bernoulli's constant are obtained for the case $\Delta=0$, as it is in our theory.

Let $s$ and $n$ be the coordinates along and normal to the layer (figure 1, with only the lower half of the eddy considered). Along the eddy boundary $s$ grows from 0 at $\mathrm{A}$ to $s_{B}$ at $\mathrm{B}$ and then along the symmetry line to $s_{A}$ at A. Let $U(s)$ be the velocity of the inviscid Sadovskii flow on the inner side of the eddy boundary and along the symmetry line. For $g=\frac{1}{2}\left(u^{2}-U^{2}\right)-\Delta$, where $u$ is the velocity component along the $s$-direction in the boundary layer, the corresponding boundary-layer problem in the Mises variables takes the form

$$
\begin{gathered}
\frac{\partial g}{\partial s}=\frac{u}{\operatorname{Re}} \frac{\partial^{2} g}{\partial \psi^{2}}, \\
g=0, \quad s=0, \quad \psi<0, \\
g(0, \psi)=g\left(s_{A}, \psi\right), \quad \psi>0, \\
\frac{\partial g}{\partial \psi}=0, \quad \psi=0, \quad s_{B}<s<s_{A}, \\
\psi \rightarrow \infty, \quad g \rightarrow-A=\text { const. }
\end{gathered}
$$


Here $\psi$ is a stream function, all variables have been normalized with the original scales (that is with the velocity at infinity and the size of the body), but only the main terms are retained in the equation and boundary conditions. Condition (2.2) is the condition in the oncoming flow whilst condition (2.3) follows from the inviscid nature of the flow in the turn region near point $A$ as explained above. The similar condition near point $\mathrm{B}$ is taken into account implicitly, as the solution of (2.1) satisfies it. Condition (2.4) is a symmetry condition and (2.5) follows from matching with the inviscid flow inside the eddy. The solution is to be found in the region $-\infty<\psi<+\infty$ for $0 \leqslant s \leqslant s_{B}$ and $0 \leqslant \psi \leqslant+\infty$ for $s_{B}<s \leqslant s_{A}$.

Boundary-value problems similar to $(2.1)-(2.5)$ are usually believed to have a solution only for a single value of $\Delta$, thus determining this value. For a heat equation this has been proved by Kolosov \& Shifrin (1975). The solution of (2.1)-(2.5) is trivial: $g(s, \psi) \equiv 0, \Delta=0$. The uniqueness of this solution was considered by Chernyshenko (1988). So assuming $\Delta \neq 0$, we have arrived at $\Delta=0$. Hence the assumption $\Delta \neq 0$ is not correct and, in fact, $\Delta=0$. Note that, from the definition of $g$, this immediately implies that $u=U(s)$.

As $\Delta=0$, on the eddy scale the flow tends to the inviscid Sadovskii flow with a zero jump in the Bernoulli constant. This is the main result of this section. Note that the only assumption necessary for its validity is that the eddy length and width are of the same order, which is greater than 1 when $R e \rightarrow \infty$, and that, as stated earlier, this assumption is validated later. Unbounded Sadovskii flows for $\Delta=0$ were numerically calculated by Sadovskii (1970) and Saffman \& Tanveer (1982), and the Sadovskii flow in a channel by Turfus (1993) and Chernyshenko (1993).

\subsection{Recirculating boundary layer for vorticity}

The eddy-scale flow has vorticity discontinuities at both the eddy boundary and the symmetry line. Hence there is a boundary layer in which the vorticity smoothly changes from the value in the eddy to zero in the external flow. As the velocity of the inviscid flow on the eddy scale is continuous, the main term of the velocity in the boundary layer is trivial and equals the velocity in the inviscid flow at the eddy boundary and symmetry line, $U(s)$. This was in fact demonstrated in the preceding section. To describe the vorticity diffusion the next term of the expansion of the velocity in the boundary layer should be considered. The same coordinate system as in $\$ 2.1$ is used here (see figure 1). In these coordinates the boundary layer must be considered inside the region shown in figure 2 . In the part of the layer near the eddy boundary $\left(0 \leqslant s \leqslant s_{B}\right)$ the coordinate $n$ in the boundary layer change from $-\infty$ to $+\infty$, while near the symmetry line $\left(s_{B}<\mathrm{s} \leqslant s_{A}\right)$, owing to the symmetry, it is sufficient to consider only $n \geqslant 0$. The anticipated velocity profiles are shown in figure 2 , based on the two leadingorder terms. At $s=0, n<0$ the velocity corresponds to the oncoming irrotational flow and is uniform. The velocity profiles for $n>0$ at $s=s_{A}$ and $s=0$ are the profiles immediately before and after the turn region near point $A$. These are expected to be identical by analogy with the analysis of the turn regions in the preceding section and a rigorous proof is given in $\$ 2.4$. Note the discontinuity in velocity at $s=0, n=0$. This discontinuity must be allowed for, because the fluid from the boundary layer adjoining the symmetry line, on entering the turn region, comes in contact with the fluid flowing from upstream, and the Bernoulli constants on the different sides of the dividing streamline may be different. The notion of the Bernoulli constant is appropriate here, because in the turn region the flow is expected to be inviscid. The velocity jump is an important feature because it is closely related to the vorticity flux originating primarily from the body. Owing to the parabolic nature of the boundary-layer equations the 


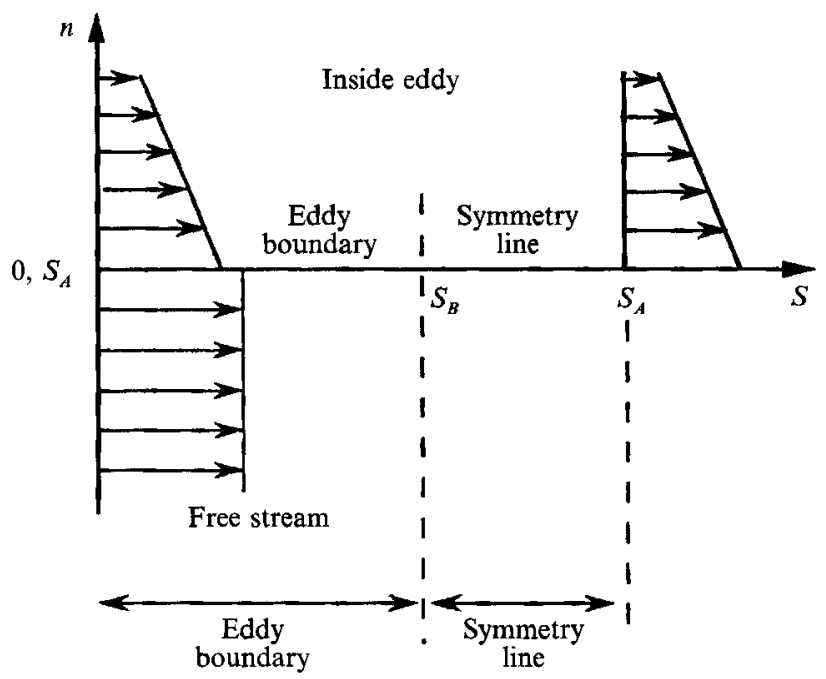

FIGURE 2. Anticipated velocity profiles near eddy boundary and on symmetry line.

condition that the velocity profile be continuous in the turn region near point $\mathrm{B}$ (i.e. that the profiles emerging from and entering the turn region should be the same) can be taken into account implicitly by considering the solution to continue across $s=s_{B}, n>0$ in figure 2 .

The governing equation and boundary conditions in the recirculating boundary layer can be formulated in terms of the velocity or vorticity. The latter form is much more convenient, as it is sufficient to consider only the main term of the vorticity expansion instead of two terms of the velocity expansion. The main term of the vorticity is governed by an equation which, in Mises variables $s$ and $\psi$ takes the form

$$
\frac{\partial \omega}{\partial s}=\frac{U(s) \partial^{2} \omega}{R e \partial \psi^{2}} .
$$

Inside the layer

$$
\psi=U(s) n
$$

Use is made here of the fact that the main term for the velocity is known. The boundary conditions are

$$
\begin{gathered}
s=0, \quad \psi<0, \quad \omega=-[B] \delta(\psi-0), \\
\psi>0, \quad \omega(0, \psi)=\omega\left(s_{A}, \psi\right), \\
\psi=0, \quad s_{B}<s<\mathrm{s}_{\mathrm{A}}, \quad \omega(s, 0)=0, \\
\psi \rightarrow \infty, \quad \omega \rightarrow \omega_{\infty} .
\end{gathered}
$$

Here again the original scales for all quantities are used and only the leading terms are retained. This formulation can be obtained by applying a usual boundary-layer approximation to the vorticity equation. In $(2.11) \omega_{\infty}$ is the value of the vorticity in the eddy and (2.10) is a symmetry condition. The condition (2.9) implies that the dependence of the vorticity $\omega$ on the stream function remains unchanged inside the turn region near point $\mathrm{A}$, and a similar condition at point $\mathrm{B}$ is implicitly accounted for by (2.6). The conservation of vorticity along streamlines inside the turn regions follows from an argument similar to that employed in the previous section and will be justified in $\$ 2.4$.

In (2.8) $[B]$ is the jump in the Bernoulli constant at $s=0, n=0$ (see again figure 2), 
because in the context of the boundary-layer approximation $\partial B / \partial \psi=-\omega$, where $B=\left(\frac{1}{2} \mathrm{u}^{2}+\mathrm{p}\right)$ and $p$ is the pressure. The pressure is continuous and the delta function in (2.8) is the derivative of a step function.

An obvious substitution transforms (2.6) into the one-dimensional unsteady heat equation. Hence the theorem proved by Kolosov \& Shifrin (1975) applies. From this theorem it follows that for given $[B], R e, U(s), s_{A}$ and $s_{B}$ the solution of (2.6)-(2.11) exists only for a certain value of $\omega_{\infty}$. Hence (2.6)-(2.11) give not only $g(s, \psi)$, but also $\omega_{\infty}$ as part of the solution. The physical nature of this result concerns a vorticity balance and was explained while deriving (1.3) in the Introduction.

The system (2.6)-(2.11) was solved numerically for $U(s)$ and $b$, see below, corresponding to the flow past an isolated body (Chernyshenko 1988) but only in order to check the more general approach developed by Chernyshenko (1982). Apart from such checking, other results of the computation did not seem very interesting at the time and were not included in that paper. Similar calculations have also been made more recently by Turfus (1991, private communication). From the results of Chernyshenko (1982, see also the Appendix) the following explicit expression for $\omega_{\infty}$ emerges:

$$
\omega_{\infty}=-2 D_{0}(b)[B]\left[R e / t\left(s_{A}\right)\right]^{\frac{1}{2}},
$$

where

$$
t(s)=\int_{0}^{s} U(s) \mathrm{d} s, \quad b=t\left(s_{A}\right) / t\left(s_{B}\right) .
$$

Function $D_{0}(b)$ is tabulated and $D_{0}(b)=D(b, 0)$ in the notation of Chernyshenko (1982), see the Appendix.

In the lower half of the eddy considered here, $\omega_{\infty}>0$ and $[B]<0$. In accordance with the definition above, $t\left(s_{A}\right)$ is the circulation of the velocity around the lower half of the eddy. Hence, by Stokes' theorem, it equals the integral of the vorticity over the lower half of the eddy. The substitution $t\left(s_{A}\right)=\frac{1}{2} \omega_{\infty} S$, where $S$ is the total area of both halves of the eddy, yields

$$
\omega_{\infty}=-2[B] D_{0}(b)\left[2 \operatorname{Re} /\left(\omega_{\infty} S\right)\right]^{\frac{1}{2}} .
$$

This is an exact form of (1.3), combined with (1.1), because, as explained in the Introduction, the jump in the Bernoulli constant equals the vorticity flux from the body. It is yet to be proved though that $[B]$ in (2.13) indeed equals the jump in the Bernoulli constant on the body scale.

It is now possible to determine the drag. Multiplying (2.6) by $\psi$ yields

$$
\psi \frac{\partial \omega}{\partial s}=\frac{U}{R e} \psi \frac{\partial^{2} \omega}{\partial \psi^{2}}
$$

This can easily be reduced to an equation similar to the unsteady heat conduction equation and it follows from the properties of the solutions of such equations (Tihonov \& Samarskii 1972) that both terms decay exponentially as $\psi \rightarrow-\infty$. Hence double integrals of both sides of this equation over the entire region in figure 2, that is over $\sigma=\left(0 \leqslant s \leqslant s_{A}, \psi>0\right) \cup\left(0 \leqslant s \leqslant s_{B}, \psi<0\right)$, converge. Now for $\psi>0$

because of (2.9) and, for $\psi<0$,

$$
\int_{0}^{s_{A}} \psi \frac{\partial \omega}{\partial s} \mathrm{~d} s=0
$$

$$
\int_{0}^{s_{B}} \psi \frac{\partial \omega}{\partial s} \mathrm{~d} s=\psi\left(\left.\omega\right|_{s=s_{B}}\right.
$$


because of (2.8). Therefore

$$
\iint_{\sigma} \psi \frac{\partial \omega}{\partial s} \mathrm{~d} s \mathrm{~d} \psi=\left.\int_{-\infty}^{0} \psi \omega\right|_{s=s_{B}} \mathrm{~d} \psi
$$

For the right-hand side we have, for $s \leqslant s_{B}$,

$$
\int_{-\infty}^{+\infty} \frac{U}{R e} \psi r \frac{\partial^{2} \omega}{\partial \psi^{2}} \mathrm{~d} \psi=\left.\frac{U}{\operatorname{Re}} \psi \frac{\partial \omega}{\partial \psi}\right|_{-\infty} ^{+\infty}-\int_{-\infty}^{+\infty} \frac{U}{\operatorname{Re}} \frac{\partial \omega}{\partial \psi} \mathrm{d} \psi=\left.\frac{U}{\operatorname{Re}} \omega\right|_{\psi=-\infty} ^{\psi=+\infty}=-\frac{U \omega_{\infty}}{\operatorname{Re}} .
$$

Here use is made of $\partial \omega / \partial \psi$ tending to zero exponentially when $\psi \rightarrow+\infty$ or $\psi \rightarrow-\infty$ (Tihonov \& Samarskii 1972), and of $\omega \rightarrow 0$ for $\psi \rightarrow-\infty$. The latter follows from (2.8) and properties of solutions of a heat equation. For $s_{B} \leqslant s \leqslant s_{A}$, analogously, we obtain by using (2.10)

$$
\int_{0}^{+\infty} \frac{U}{R e} \psi \frac{\partial^{2} \omega}{\partial \psi^{2}} \mathrm{~d} \psi=\left.\frac{U}{R e} \psi \frac{\partial \omega}{\partial \psi}\right|_{0} ^{+\infty}-\int_{0}^{+\infty} \frac{U}{R e} \frac{\partial \omega}{\partial \psi} \mathrm{d} \psi=-\frac{U \omega_{\infty}}{R e}
$$

Therefore

$$
\iint_{\sigma} \frac{U}{R e} \psi r \frac{\partial^{2} \omega}{\partial \psi^{2}} \mathrm{~d} \psi=-\frac{\omega_{\infty}}{R e} \int_{0}^{s_{A}} U(s) \mathrm{d} s=-\frac{\omega_{\infty}^{2}}{R e} \frac{S}{2}
$$

where again Stokes' theorem has been used. Equating the double integrals of the leftand right-hand sides we arrive at

$$
\left.\int_{-\infty}^{0} \psi \omega\right|_{s=s_{B}} \mathrm{~d} \psi=-\frac{\omega_{\infty}^{2} S}{2 R e} .
$$

The fluid at $s=s_{B}, \psi<0$ enters the downstream turn region and emerges to the wake with the same $\omega(\psi)$ owing to the anticipated effectively inviscid nature of the flow in this region. Then the fluid moves downstream in the wake. The flow in the wake is governed by an equation of the same form as (2.6), with $U(s)$ equal to the velocity of the Sadovskii flow along the symmetry line behind the eddy. Multiplying the governing equation by $\psi$ and integrating with respect to $\psi$ from $\psi=-\infty$ to 0 it is quite easy to deduce that

$$
\int_{-\infty}^{0} \psi \omega \mathrm{d} \psi
$$

remains constant in the wake. Far downstream in the wake the velocity tends to 1 and hence $\psi$ tends to $y$. A boundary-layer approximation gives $\omega=-\partial u / \partial y$.

Hence

$$
\begin{aligned}
-\frac{\omega_{\infty}^{2} S}{R e}=\int_{-\infty}^{+\infty} \psi \omega \mathrm{d} \psi=-\int_{-\infty}^{+\infty} y \frac{\partial u}{\partial y} \mathrm{~d} y=-\int_{-\infty}^{+\infty} y \frac{\partial(u-1)}{\partial y} \mathrm{~d} y \\
=-\left.y(u-1)\right|_{-\infty} ^{+\infty}+\int_{-\infty}^{+\infty}(u-1) \mathrm{d} y=\int_{-\infty}^{+\infty}(u-1) \mathrm{d} y=-G .
\end{aligned}
$$

Here $G$, by the usual expression resulting from integration of the velocity profile in the far wake where the velocity is close to that at infinity, is the drag normalized by the density, length and velocity scales. Therefore

$$
c_{d}=\omega_{\infty}^{2} S / R e,
$$

where $c_{d}=\operatorname{drag} /\left(\rho U_{\infty}^{2} R\right), \rho$ is the density and $R$ is an appropriate lengthscale, with $R e=U_{\infty} R / \nu$. This calculation is somewhat lengthy and the same result can be 
obtained more briefly by calculating the rate of energy dissipation. According to the Bobylev-Forsythe formula (Serrin 1959) the non-dimensional rate of energy dissipation equals $(1 / R e) \int \omega^{2} \mathrm{~d} x \mathrm{~d} y$, where the integral is taken over the entire flow field. This leads directly to (2.14), if the influence of the vorticity distribution in the regions not considered so far (i.e. those discussed in \$\$2.3-2.6) may be neglected. This will be justified only after the analysis of these other regions has been completed. Therefore the calculation via the far wake, as given above, is more rigorous at this stage of the investigation.

Although the eddy length is not yet determined, the value of

$$
C=\omega_{\infty}^{2} S
$$

can be found for a given $H / L$ from Sadovskii flow calculations, because $S$ varies directly as the square of the eddy length and, for the upstream velocity held constant, $\omega_{\infty}$ varies inversely with the eddy length. Hence (2.14) may be written as

implying that $C=C(H / L)$.

$$
c_{d}=C / R e,
$$

\subsection{The body-scale flow}

As will be seen later, the flow on the body scale cannot be matched directly with the eddy-scale flow; there are several distinguishing regions between them. Nevertheless, it is convenient to discuss the body-scale flow at this stage in order to determine the main characteristics of the flow. Regions having other scales are required only to prove the self-consistency of the theory. It will be shown later that the body-scale flow matches a flow on a somewhat larger scale - the intermediate region - which in turn matches a still larger cusp region; the latter matches the turn region. The flows in all these regions are considered in the following sections and found to be effectively inviscid. For this reason Bernoulli's (constant) jump is the same in all these regions and equals $[B]$. (Note that $[B] \neq \Delta=0$, as they are parameters of the flows on different scales.) In $\S 2.5$ it is shown that on a certain scale, which is named a cusp scale, the eddy boundary has a cusp. Consequently the reversed flow in the eddy on this scale quickly slows down as it approaches the body. As a result, on smaller scales the fluid in the eddy is effectively stagnant. These properties of the flows in other regions may be considered as temporary assumptions and will be justified later.

The only flow on the body scale meeting the conditions outlined, that is that the fluid in the eddy is stagnant and the Bernoulli's constant jump across the eddy boundary equals $[B]$, is the Kirchhoff flow with the value of the velocity on the free streamline $V_{1 \infty}=(-2[B])^{\frac{1}{2}}$. Note that according to the notation in the previous section, where the lower half of the eddy was considered, $[B]$ is negative. The drag coefficient is therefore given by the formula

$$
c_{d}=k_{d} V_{1 \infty}^{2}=-2 k_{d}[B],
$$

where $k_{d}$ is the drag coefficient in the Kirch hoff flow with velocity on the free streamline equal to unity (as in $\$ 1$ ). Comparison with (2.14), in view of (2.15), yields $-[B]=C /\left(2 k_{d} R e\right)$ and this allows $V_{10}$ to be calculated. Since the Kirchhoff flow is an inviscid one, for consistency the effective Reynolds number, $R e_{b}$, on the body scale must tend to infinity. This is indeed so because $\operatorname{Re}_{b}=\operatorname{Re} V_{1 \infty}=\left(\mathrm{CRe} / k_{a}\right)^{\frac{1}{2}} \rightarrow \infty$.

There is no need to give a detailed description of the flow on the body scale and other smaller scales inside the body scale since an excellent description has in fact already been given by Smith (1979). To apply his results to the body-scale flow in our theory it is sufficient to renormalize them. This may be done by substituting our value of the free-streamline velocity $V_{100}$ instead of that used by Smith. Considering $V_{100}$ as the 
velocity scale in the body-scale flow, the Smith (1979) formulae concerning the bodyscale flow may be used by substituting $R e_{\text {Smith }}=R e_{b}=V_{1 \infty} R e$. Smith showed that the limiting flow past a smooth body such as a circular cylinder satisfies a condition of smooth separation and this gives a unique value for $k_{d}$. For bodies with sharp edges, $k_{d}$ is determined by the condition that separation occurs at the edge.

The four equations (2.13), (2.15)-(2.17) allow the four unknowns, $\omega,[B], S$ and $c_{d}$, to be determined, with results which can be written in the following form:

$$
\begin{gathered}
\omega_{\infty} k_{d}^{2} R e=2 C D_{0}^{2}(b), \\
-[B] k_{d} R e=\frac{1}{2} C, \\
S /\left(k_{d}^{4} R e^{2}\right)=1 /\left[4 C D_{0}^{4}(b)\right],
\end{gathered}
$$

and $c_{d}$ is given by (2.16). Quantities on the right-hand sides of (2.18)-(2.20) and $C$ in (2.16) depend only on the Sadovskii flow and for the flow past an isolated body they are constant. For the cascade flow considered here they depend on the ratio $H / L$. All other Sadovskii flow characteristics also depend on $H / L$. In particular we may denote $S / L^{2}=\alpha(H / L)$ and then from (2.20) it immediately follows that

$$
L /\left(k_{d}^{2} R e\right)=1 /\left[2 D_{0}^{2}(b)(\alpha C)^{\frac{1}{2}}\right] .
$$

Multiplying this expression by $H / L$ we get

$$
H /\left(k_{d}^{2} R e\right)=H /\left[2 L D_{0}^{2}(b)(\alpha C)^{\frac{1}{2}}\right] .
$$

The right-hand side of (2.22) is a function of $H / L$ only. Hence, for a specified value of $H /\left(k_{d}^{2} R e\right),(2.22)$ gives the value of $H / L$ and consequently the values on the left-hand sides of $(2.18)-(2.21)$. This theory therefore gives the asymptotics for $R e \rightarrow \infty, H / R e=$ const. To obtain quantitative results it is more convenient to consider (2.18)-(2.22) as relations between $H /\left(k_{d}^{2} R e\right)$ and all other quantities written in a parametric form, with $H / L$ being the parameter.

\subsection{The turn regions}

Within the framework of the method of matched asymptotic expansions an asymptotic theory is believed to be true if it is self-consistent. This means that the expansions in all distinguished limits are considered and can be properly matched. The assumptions made above concerning, first, the effectively inviscid character of the flows in the turn regions and on the scales between the body scale and the recirculating-layer scale and, second, the fluid in the eddy being stagnant on the smaller scales, have yet to be justified. Proving both the validity of these assumptions and the self-consistency of the theory is the main purpose of this and the two following sections. Note that since the turn regions near points A and B are similar; they are considered simultaneously and the differences will be pointed out subsequently.

The lengthscale of the recirculating layer is the same as that of the eddy, which was found above to be of order $R e$, and the velocity is the same order (unity) as that at infinity. Therefore, the characteristic thickness of the recirculating layer which, as usual for boundary layers, equals the layer length divided by the square root of the Reynolds number based on this length, is also of order 1 . This estimate is not valid near points $A$ and $B$ since the velocity decreases near these points, leading to an increase in the layer thickness. Indeed, the order of magnitude of the flow rate in the layer near A and $\mathrm{B}$ and far from these points should be the same. The leading term for the velocity in the layer was shown in $\S 2.1$ to be $U(s)$. Hence near points $\mathrm{A}$ and B the layer thickness is proportional to $U^{-1}(s)$ and $U^{-1}\left(s-s_{B}\right)$, respectively. The lengthscales in the orthogonal directions in the turn regions must be equal, otherwise the corresponding 
limit would not be a distinguished one, because all the leading terms in the equations of motion in the turn region would be contained in the boundary-layer equations of the recirculating layer. Therefore we arrive at the estimate $l_{t} \sim U^{-1}\left(l_{t}\right)$, where $l_{t}$ is the lengthscale of the turn regions. The leading term of the asymptotics of $U(s)$ for $s \rightarrow 0$ in the Sadovskii flow, obtained by Sadovskii $(1971 b)$ and also by Saffman \& Tanveer (1982), can be expressed as $U(s) \sim\left(s \omega_{\infty}\right) \ln \left(s \omega_{\infty}\right)$. Taking into account $(2.18)$ for $H / L$ fixed we obtain the estimate $l_{t} \sim(\operatorname{Re} / \ln R e)^{\frac{1}{2}}$. Accordingly, the velocity scale in the turn regions is $U_{t} \sim l_{t}^{-1} \sim(\ln R e / R e)^{\frac{1}{2}}$.

As is easily seen from these results for $l_{t}$ and $U_{t}$, the Reynolds number formed from the length and velocity scales in the turn regions $R e \rightarrow \infty$. Therefore the flow in these regions is effectively inviscid. The vorticity in the turn regions is determined by matching with the recirculating boundary layer and hence has the same order as the vorticity there and the vorticity in the eddy (the latter two being of the same order because of (2.11)). Therefore, in view of (2.18)-(2.19), in the turn regions $[B] / U_{t}^{2} \sim 1 / \ln$ $R e \rightarrow 0$ and $\omega_{t} l_{t} / U_{t} \sim 1 / \ln R e \rightarrow 0$. For this reason the velocities produced by Bernoulli's constant jump and by the vorticity may be neglected in the leading term of the expansion. Hence, the leading term here is a potential flow without a jump in the Bernoulli constant. The next term is only $1 / \ln R e$ times less and takes into account the vorticity and discontinuity of Bernoulli's constant. In the external Sadovskii flow, in accordance with the asymptotics mentioned above, $\psi=C_{2} \operatorname{Im}(z / R e)^{2} \ln (z / R e) \operatorname{Re}$ for $z / \operatorname{Re} \rightarrow 0$, where $z=x+\mathrm{i} y$ and $x, y$ are Cartesian coordinates. The flow in the turn region, which can be matched with this one, is a potential flow with a stagnation point. The stream function for such a flow is $\psi_{t}=C_{1} \operatorname{Im}\left(z / l_{t}\right)^{2}, C_{1}=$ const. These expansions are easily matched in the intermediate limit $z \sim R e^{\frac{1}{2}}$ if $C_{1}=-C_{2}$.

The turn region near point $B$ differs from that near $A$ mainly by the absence of the discontinuity of Bernoulli's constant. So in the rear part of the eddy there is no cusp similar to that considered below. This feature can be easily seen in the numerical results of Fornberg (1985). Note the role played by the logarithmic factor in the Sadovskii flow asymptotics near the stagnation point. Were it not for this factor, there would be only a single region combining the features of the cusp (see below) and the turn regions.

It is very easy to verify that, owing to the effectively inviscid character of the flow, the leading term for the vorticity in the turn regions is governed by the equation $\boldsymbol{u} \cdot \boldsymbol{\nabla} \omega=0$, which leads immediately to the conservation of vorticity along streamlines. This validates the condition (2.8) and the similar implicit condition near point $B$ in the recirculating layer. Note that although the expansion in the turn region is built in powers of $\ln R e$, the condition (2.8) has far greater accuracy because, since the flow in the turn region is inviscid, it holds true until the viscous forces, which in this region are of order $1 / R e_{t} \sim 1 / R e$, manifest themselves. The conservation of the jump $[B]$ in the Bernoulli constant inside the forward turn region follows for the same reasons.

\subsection{The cusp region near $\mathrm{A}$}

The flow in the turn region slows down as it approaches the stagnation point, while the jump in the Bernoulli constant remains the same. Hence the expansion in the turn region is not valid near point $A$, where $[B]$ and the square of the velocity are of the same order. Therefore, another distinguishing limit should be introduced. As the velocity in the potential flow near the stagnation point inside the turn region behaves as $U_{t}\left(x^{2}+y^{2}\right)^{\frac{1}{2}} / l_{t}$, the length and velocity scales $l_{c}$ and $U_{c}$ in this region satisfy the relation $U_{c} \sim U_{t} l_{c} / l_{t} \sim(-[B])^{\frac{1}{2}} \sim 1 / \operatorname{Re}^{\frac{1}{2}}$ and therefore $l_{c} \sim l_{t}(\ln R e)^{\frac{1}{2}} \sim(\operatorname{Re} / \ln \operatorname{Re})^{\frac{1}{2}}$. Hence the Reynolds number $R e_{c}=U_{c} l_{c} R e=R e / \ln R e \rightarrow \infty$ and the flow is effectively inviscid. The stream-function scale is $\Psi \sim U_{c} l_{c} \sim(\ln R e)^{-1}$. 
The vorticity inside the eddy in the cusp region is specified by matching with that in the turn region, which in turn matches with that in the recirculating layer. Inside the turn region the vorticity is constant along streamlines, as shown in the preceding section. Owing to the condition $\omega=0$ on the symmetry line, in the recirculating layer entering from behind the forward turn region $\omega(\psi) \sim$ const $\psi / \operatorname{Re}$ for $\psi \rightarrow 0$. The factor $1 / R e$ appears because the vorticity in the recirculating boundary layer is of order $1 / R e$, as follows from (2.11) and (2.18). For this reason the vorticity scale $\Omega_{c} \sim \Psi_{c} / R e=1 /(\operatorname{Re} \ln R e)$. The velocity induced by the vorticity distribution in this region is much smaller than the velocity scale: $\Omega_{c} l_{c}=1 /\left(R^{\frac{1}{2}}(\ln R e)^{2}\right) \ll 1 / \operatorname{Re}^{\frac{1}{2}} \sim U_{c}$. Therefore the flow here is also potential. The flow outside the eddy is potential because of the upstream boundary conditions. Thus we arrive at the conclusion that the flow in this region is a collision of two potential flows with different Bernoulli constants. Such a flow is a special case of the flow considered by Lukerchenko (1990) and the dividing streamline in such a flow has a cusp. The possibility of matching this flow with the turn region is evident.

Conservation of vorticity and the Bernoulli-constant jump along streamlines in the cusp region follows from the effectively inviscid nature of the flow and the same remark on accuracy as that made at the end of the previous section applies.

The cusp considered here should not be confused with the cusp in Sadovskii's flow with non-zero jump in the Bernoulli constant. The Sadovskii flow on the eddy scale in this theory has no cusp because the Bernoulli-constant jump is zero. The existence of a cusp near the body on the scale considered in this section is important, because it causes the velocity inside the eddy to tend to zero sufficiently quickly that on the smaller scales the fluid in the eddy is effectively stagnant, as was assumed in $\$ 2.3$.

\subsection{The intermediate region}

The flows on the body scale and cusp scale cannot be matched, because in the Kirchhoff flow the width of the eddy is of order $x^{\frac{1}{2}}$ for $x \rightarrow \infty$ and in the cusp flow it is of order $x^{\frac{3}{2}}$ for $x \rightarrow 0$ (see, for example, Smith 1985b). Therefore an intermediate region must be introduced. The velocity scale in this region is determined by $[B]$ and is the same as in the body-scale and cusp flows. As the lengthscale here is larger than the body scale, the flow is effectively inviscid in the intermediate region as well. In this region the velocity inside the eddy is asymptotically small in comparison with the velocity outside, owing to the existence of a cusp on the larger scale.

It is possible to construct the solution in the intermediate region in the usual way by considering the internal structure of this region. This is straightforward but lengthy. Another, briefer approach is followed in this paper by considering potential separated flow with free streamlines past a plate near a wall (figure 3). Such a flow exists and its complex velocity can be found explicitly from the expression

$$
\frac{\mathrm{d} w}{\mathrm{~d} z}=\left\{1-\left[\frac{1}{2}+\mathrm{i}\left(a^{2}-\left(1 / w-\left(a^{2}+\frac{1}{4}\right)^{\frac{1}{2}}\right)^{2}\right)^{\frac{1}{2}}\right]^{-1}\right\}^{\frac{1}{2}},
$$

where $a$ is an arbitrary parameter, $w$ is the complex velocity potential and $z=x+\mathrm{i} y$. It is easy to verify that for $a \rightarrow \infty$ the ratio of the plate length to its distance to the wall tends to zero. The flow on the plate scale tends to the Kirchhoff flow and on the scale of distance to the wall tends to the flow with a cusp. The distance from the plate to the wall is of order $a$, the plate length is of order $a^{-3}$, and the size of the intermediate region, the existence of which may be checked directly, is of order $a^{-1}$. There are no essential differences between matching the regions of the flow in figure 3 and matching the corresponding regions in our theory. The intermediate region has the same 


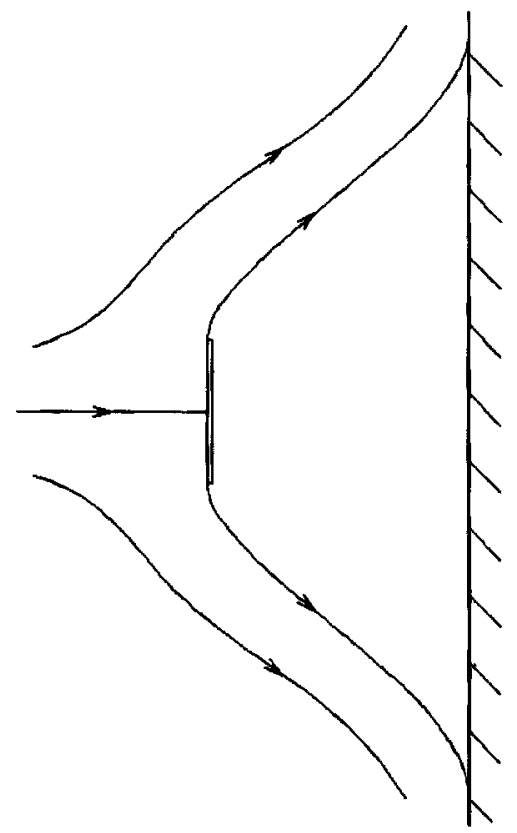

Figure 3. Model flow. A normal flat plate just upstream of an infinite wall.

structure in both cases. Choosing the proper lengthscale of the flow in figure 3 and the value of $a$, it is easy to find that the length of the intermediate region in our theory is

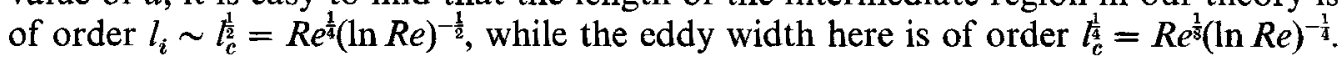

Some of the distinguishing limits have not yet been considered. For example, at the eddy boundary in the intermediate and cusp regions there is a viscous mixing layer due to the discontinuity of the velocity in the inviscid flow. It continues the mixing layer on the body scale and is continued downstream into the upstream turn region, although only as a second-order team in the velocity expansion. The detailed description of these mixing layers, being quite obvious, is uninteresting and for brevity is not given here. For the self-consistency of our theory it is essential that the width of these mixing layers be much smaller than the width of the corresponding inviscid regions. This is readily seen from the orders of magnitude of the eddy width and lengthscales given above. In the inviscid flow inside the downstream turn region there is a discontinuity of the vorticity on the symmetry line. Hence, a viscous sublayer must be introduced. Again, being of a trivial nature, it will not be discussed further.

The proof of the self-consistency of our theory has now been completed.

\section{The asymptotics for $H / R e \rightarrow 0$}

The theory for the flow past an isolated body (Chernyshenko 1988) is clearly a special case of the present theory, in which $b, C$ and $\alpha$ assume values specific to the Sadovskii flow in an unbounded domain. To establish the whole region of validity it is necessary to investigate the asymptotics of this theory for $H / R e$ (see (2.22)) tending to zero.

The asymptotic behaviour of the solution crucially depends on the asymptotics of the Sadovskii flow for $H / L \rightarrow 0$. Numerical calculations by Chernyshenko (1993) show that in this limit the ratio of the eddy half-width, $W$, to $H$ approaches a constant value close to $\frac{2}{3}$. The eddy boundary has been found to be almost flat for small $H / L$ except 
in the vicinity of the ends of the eddy. The stream function in the Sadovskii flow (for which, again, only the upper half of the eddy is considered) satisfies the equation

$$
\begin{array}{ll}
\nabla^{2} \psi=0, & \psi>0, \\
\nabla^{2} \psi=-\omega=\mathrm{const}, & \psi \leqslant 0 .
\end{array}
$$

The derivative with respect to $x$ drops out of this equation over most of the eddy length in the limit $H / L \rightarrow 0$ and the resulting equation can be solved easily with the result

$$
\left.\begin{array}{ll}
\psi=H(y-W) /(H-W), & y>W, \\
\psi=-\frac{1}{2} \omega y(y-W), & 0 \leqslant y \leqslant W .
\end{array}\right\}
$$

The velocity at upstream infinity is assumed here to be equal to 1 in accordance with the velocity scale used throughout this paper. The jump in the Bernoulli constant across the eddy boundary, that is at $y=W$, equals zero provided that $\omega=-2 H /[W(H-W)]$. The remaining undetermined parameter $W$ cannot be found without considering the flow in the vicinity of the ends of the eddy, where the eddy boundary is not flat. Fortunately, however, the complete solution of this problem is not necessary for our purpose, although it has been found numerically by Chernyshenko (1993). The requirement of momentum conservation allows $W$ to be found without solving this problem in full. Momentum conservation can be expressed in the following form:

$$
I(x)=\int_{0}^{H}\left(u^{2}+p\right) \mathrm{d} y=\text { const },
$$

where $I(x)$ is the momentum flux, $p$ is the pressure and, as before, the density is taken as unity for convenience. Far from the ends of the eddy the pressure is uniform. In the middle of the eddy the velocity $u$ is readily calculated from (3.1) and far from the eddy $u=1$. Equating the momentum flux in the middle part of the eddy and far downstream we get one equation with two unknowns: $W$ and the difference between the pressures in the middle part of the eddy and far from it. Bernoulli's equation supplies the second relation needed to find these two unknowns. The solution of these equations gives $W / H=\frac{2}{3}$; the corresponding value of $\omega$ equals $-9 / H$ and $9 / H$ in the upper and lower halves of the eddy, respectively.

Note that as $H / L \rightarrow 0$, three regions emerge in the Sadovskii flow. The separation region and the reattachment region have a lengthscale $H$ in both directions and the main part of the eddy has a lengthscale $L$ in the $x$-direction and $H$ in the $y$-direction. A more detailed account of this flow, including its asymptotic behaviour for $H / L \rightarrow 0$, is given by Chernyshenko (1993).

Now it is quite easy to deduce that for $H / L \rightarrow 0$

$$
C \rightarrow 108 L / H, \quad b \rightarrow 0.5, \quad \alpha \rightarrow 4 H /(3 L) .
$$

Substituting these asymptotics in (2.16), (2.18)-(2.22) we obtain

$$
\begin{gathered}
{[B] \rightarrow-\frac{9}{4} \frac{k_{d}}{D_{0}^{2}(0.5)} \frac{1}{H} \approx-45.7 \frac{k_{d}}{H},} \\
c_{d} \rightarrow \frac{9}{2} \frac{k_{d}^{2}}{D_{0}^{2}(0.5)} \frac{1}{H} \approx 91.3 \frac{k_{d}^{2}}{H}, \\
L \rightarrow \frac{1}{24} \frac{k_{d}}{D_{0}^{2}(0.5)} R e \approx 0.846 k_{d}^{2} R e .
\end{gathered}
$$


It is remarkable that the first three, (3.2)-(3.4), do not depend on $R e$ and $L$ does not depend on $H$.

To find the region of validity of the theory it is necessary to find the length and velocity scales of the main flow regions for $H \ll R e$. On the eddy scales the velocities remain of order 1 . The eddy length is of order $R e$ as is readily seen from (3.5), but the eddy width is of order $H$. Near the ends of the eddy the appropriate lengthscale in the Sadovskii flow is now $H$ rather than $R e$, in accordance with the asymptotics of the Sadovskii flow. With these orders of length and velocity (i.e. $H$ and 1) the recirculating boundary-layer thickness remains of order 1. As follows directly from the analysis in $\S 2$, the length and velocity scales in the turn, cusp and intermediate regions depend on the recirculating-layer thickness, the jump $[B]$ in the Bernoulli constant and the asymptotics of the Sadovskii flow in the vicinity of the stagnation points, which is determined by the value of the vorticity in the eddy. For $H / L \rightarrow 0$ the vorticity and the jump in Bernoulli's constant are given by (3.3). This yields the following estimates:

$$
\begin{gathered}
l_{t} \sim(H / \ln H)^{\frac{1}{2}}, \quad U_{t} \sim(\ln / H)^{\frac{1}{2}}, \quad R e_{t} \sim R e, \\
l_{c} \sim H^{\frac{1}{2}} / \ln H, \quad U_{c} \sim 1 / H^{\frac{1}{2}}, \quad \operatorname{Re} e_{c} \sim \operatorname{Re} / \ln H, \\
l_{i} \sim H^{\frac{1}{4}} /(\ln H)^{\frac{1}{2}}, \quad U_{i} \sim 1 / H^{\frac{1}{2}}, \quad \operatorname{Re} e_{i} \sim \operatorname{Re} /\left(H^{\frac{1}{4}} \ln H\right) .
\end{gathered}
$$

Here $l$ is the lengthscale, $U$ is the velocity scale and the subscripted $R e$ is the effective Reynolds number. Subscripts $t, c$ and $i$ denote turn, cusp and intermediate regions, respectively. Note that these expressions are valid only for $H \ll R e$, which corresponds to $H \ll L$. The flow on the body scale depends largely on the jump $[B]$ in Bernoulli's constant. As $[B]$ is of order $1 / H$, the velocity on the body scale is of order $1 / H^{\frac{1}{2}}$, and the effective Reynolds number $R e_{b} \sim R e / H^{\frac{1}{2}}$.

The validity of this theory depends on the following conditions.

(i) The flows on all scales from the body scale to the eddy scale must be inviscid except in the thin layer regions. This is the case as long as the following inequalities hold true: $R e_{b} \gg 1, R e_{i} \gg 1, R e_{e} \gg 1, R e_{t} \gg 1$, and the effective Reynolds number on the eddy scale $\operatorname{Re}_{e} \sim \operatorname{Re} H \gg 1$.

(ii) The lengthscales of the enclosed regions must be in the proper order:

$$
1 \ll l_{i} \ll l_{c} \ll l_{t} \ll H .
$$

(iii) The recirculating boundary-layer thickness must be much smaller than the eddy width. This means that $1 \ll H$.

All these conditions are fulfilled provided that $H \gg 1$. The reasoning for $H=O(R e)$ in $\S 2$ can now be repeated for any $H \gg 1$. Therefore this theory, created originally for $\operatorname{Re} \gg 1, H \sim L$ or, equivalently, $H \sim R e$, holds in fact for $\operatorname{Re} \gg 1, H \gg 1$. Naturally the $H \sim R e$ case is the most general.

\section{Implications for the flow with $H \sim 1$}

Although our theory is not valid for $H \sim 1$, it is possible to deduce from it a tentative asymptotic structure for this case. According to (3.5) the eddy length for $1 \ll H \ll R e$ does not depend on $H$ and is of order Re. Now it is possible to match the expansions of $L(H, R e)$ for $H / R e=\mathrm{const}, \operatorname{Re} \gg 1$ and for $H=\mathrm{const}, \operatorname{Re} \gg 1$, because the former expansion is valid for any $H \gg 1$. This matching gives $L$ proportional to $R e$, at least for sufficiently large but constant $H$ (i.e. $H=O(1)$ as $R e \rightarrow \infty$ ), so it is reasonable to expect that for $H \sim 1$ the eddy length is of order $R e$. Condition (iii) in $\S 3$ is not satisfied and the boundary layer covers the entire width of the eddy. The corresponding region 
is naturally expected to be governed by boundary-layer equations. As the viscous forces are not negligible throughout the closed-streamlines region, the PrandtlBatchelor theorem is not applicable (in contrast to the situation in the previously considered eddy-scale flow, $\S \S 2.1$. and 2.2.).

For $H \sim 1$ the scales in the turn, cusp and intermediate regions given by (3.6)-(3.8) are the same and are equal to the body scale. Therefore instead of these several regions a single body-scale region $(x=O(1), y=O(1))$ emerges, which directly matches the eddy scale. From (3.4) it follows that for $H \sim 1$ the jump in the Bernoulli constant on the body scale and the drag coefficient are finite and independent of $R e$. Accordingly, the velocity here is of order 1 and hence the flow is effectively inviscid. Far downstream on the body scale the flow must become independent of $x$, otherwise it would not match the eddy-scale flow. Outside the eddy the flow is potential because of the upstream boundary conditions. Clearly, the Euler equations governing the flow on this scale do not give the vorticity distribution inside the eddy: this is determined by matching with the reversed flow on the eddy scale. The position of the separation point is determined by the condition of a smooth separation (as in Smith 1979) or by a sharp edge. With the separation point given, the free-streamline Kirchhoff flow is determined uniquely. By analogy, the same can be expected for non-zero vorticity in the eddy. Along with Bernoulli's theorem, this provides an initial condition for the eddy-scale flow governed by the boundary-layer equations. This initial condition is discussed below and is quite similar to that proposed by Milos \& Acrivos (1986).

As mentioned above, for $H / L \rightarrow 0$ three regions emerge in the Sadovskii flow. For $H \sim 1$ the region near the forward stagnation point merges with the forward turn region, the cusp and intermediate region into a single body-scale region. Analogously, the region near the rearward stagnation point merges with the rearward turn region into a single sudden reattachment region. This region is also inviscid and has a lengthscale of order 1 in both the $x$ - and $y$-directions: $x-x_{r}=O(1), y=O(1)$. The coordinate of the reattachment $x_{r}$ is of order $R e$. The possibility of a sudden inviscid reattachment was mentioned by Milos \& Acrivos (1986) but dismissed as being unlikely.

The flow on the eddy scale can be now described as a flow governed by the boundary-layer equations and possessing two discontinuities at $x=0$ and $x=x_{r}=O(R e)$. These discontinuities represent the body scale and the sudden inviscid reattachment scale flows which, having a lengthscale in the $x$-direction of order 1 , shrink on the longer $x=O(R e)$ eddy scale. Upstream of the discontinuity which represents the body-scale flow, the flow on the eddy scale is uniform. Across the first discontinuity $(x=0)$ the Bernoulli theorem applies. It also holds true for the streamlines of the reversed flow entering the discontinuity from $x>0, y<\frac{1}{2} W_{i n}$ and emerging to $x>0, W_{i n}>y>\frac{1}{2} W_{i n}$, where $W_{i n}$ is the initial half-width of the eddy on the eddy scale. With the given vorticity in the reversed flow entering the discontinuity, the jump in the Bernoulli constant emerging from the discontinuity (and the value of $W_{i n}$ ) is determined owing to the uniqueness of the solution on the body scale, as discussed above. Bernoulli's theorem holds also across the second discontinuity and along the streamlines entering the discontinuity and then emerging from it as a reversed flow. Downstream of the second discontinuity there is no reversed flow, although the velocity profile is not yet uniform.

A very important and not very obvious feature is an additional condition on the velocity profile in the boundary layer at reattachment, following from momentum conservation combined with Bernoulli's equation in the reattachment region. This condition was used above to find the vorticity and the width of the very long eddy in 
the Sadovskii flow and could possibly be used also to locate the position $x_{r}$ of the reattachment region on the long eddy scale. Naturally, the propositions made in this section are preliminary. No definite conclusions can be reached until the solution is found completely.

\section{Comparisons and discussion}

\subsection{Some notes on the earlier theories for the flow past an isolated body}

The first theory in which the length and width of the eddy were of order $R e$ was that of Taganov $(1968,1970)$ and the flow structure in his theory is very similar to ours. Taganov assumed the jump in the Bernoulli constant across the eddy boundary to be zero and this allowed him to find the formula for the drag, which is identical to our result. In fact, the well-known Sadovskii calculations were done at Taganov's request. Unfortunately, in the subsequent papers (Taganov 1973; Bukovshin \& Taganov 1976) Taganov made several other, less appropriate, assumptions. He did not consider the turn, cusp and intermediate regions and used a mixing layer as a substitute for the recirculating layer. To complete the theory, he also used his idea of a 'second dissipative layer', the discussion of which is far beyond the scope of this paper. Taganov's quantitative results for the eddy length and other quantities, except the drag, differ from ours. Some other details concerning his theory may be found in Chernyshenko (1988). Taganov did not use modern asymptotic techniques, and his theory went unrecognized for a long time. Nevertheless, he should be credited for being the first to introduce the idea of a wide wake, the conclusion of zero jump in the Bernoulli constant across the eddy boundary, and the formula for the drag.

Peregrine (1985) and Smith (1985b) have also proposed models with a wide wake of width and length of order $R e$. Their theories were less advanced than that of Taganov and no quantitative results were obtained. The possibility of a zero jump in the Bernoulli constant was not excluded in these papers, but no special emphasis was put on this particular case. Implying the non-zero jump in the Bernoulli constant on the eddy scale, Smith (1985b) pointed out that the intermediate or 'buffer' zone of the kind considered in $\$ 2.6$ is necessary for matching the cusp and the Kirchhoff flow, but the possibility of constructing such a zone was not proved.

For completeness the proposals of a long narrow wake made by Acrivos et al. (1965, 1968), Smith (1979) and Sychev (1967) should be mentioned. The last two of these are essentially the same theory, but developed independently, and none of them has proved entirely successful (see Smith 1985b). Nevertheless, it should be pointed out that Smith's (1979) description of the body-scale flow, on renormalization, is used in this paper. Fortuitously, Smith's (1979) quantitative result for the eddy length almost coincides with ours, but all other quantities are quite different.

Objections to the well-known Batchelor model (Batchelor 1956) are also worth mentioning. Those of a physical nature have been alluded to by Smith (1979). Chernyshenko (1991) proved that except for some very unusual cases, singular points inevitably appear in the recirculating boundary layer implied by the Batchelor model. The conclusion that the Batchelor model is unlikely to be applicable for flows past bluff bodies was also made by Chernyshenko (1984) on the basis of an analysis of the pressure distribution on the body surface upstream of the separation point. A similar observation in the case of flow over a cavity was made by Herwig (1982). 


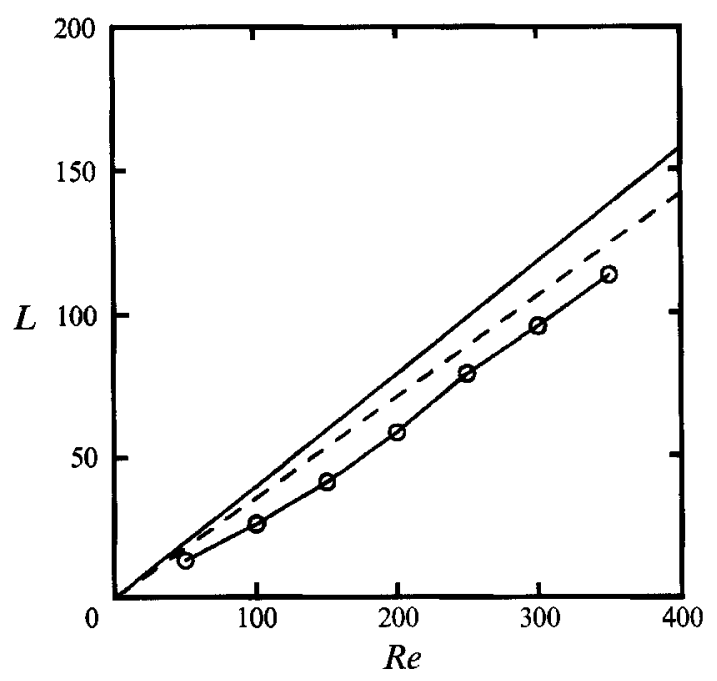

FIGURE 4. The comparison for the flow past an isolated body. The solid line is the eddy length given by (5.2). The dashed line is $L=0.354 R e$, which corresponds to $k_{d}=0.45$. Points are Fornberg's (1991) numerical results.

\subsection{Comparison with numerical calculations of the flow past an isolated body}

For $H / L=\infty$ the values of $C, \alpha$ and $b$ are the parameters of the Sadovskii flow with $\Delta=0$ (see Sadovskii 1970). The discrepancy between data for $\Delta=0$ and $\Delta \rightarrow 0$, reported by Sadovskii $(1970,1973)$, was not confirmed later, whereas data for $\Delta=0$ in the more recent work of Sadovskii \& Kozhuro (1977) are the same as for $A \rightarrow 0$ in the previous works. Sadovskii's results agree with the results of Moore, Saffman \& Tanveer (1988) and Chernyshenko (1993). From the data of Sadovskii it follows that $\alpha=0.433, b=0.542, C=\omega_{\infty}^{2} S=74.9$ and $W / L=0.300$ ( $W$ is the half-width of the eddy). Extrapolating Chernyshenko's (1993) data for flow in a channel to the unbounded case yields $\alpha=0.44, C=73, b=0.55$. On the whole the accuracy here is believed to be about $2 \%$. Both the interpolation of previous data (Chernyshenko 1982) and new calculations (Chernyshenko 1988) yield $D_{0}(0.542)=0.235$ with about $1 \%$ accuracy. These values are the same for bodies of arbitrary cross-section since the asymptotics depend on the body shape only via $k_{d}$. For the circular cylinder in Kirchhoff flow with smooth separation $k_{d}=0.50$ (Smith 1979). Hence for the flow past an isolated circular cylinder

$$
\begin{gathered}
c_{d}=74.9 R e^{-1}, \\
L=0.393 R e, \\
\omega_{\infty}=33.1 R e^{-1}, \\
{[B]=-74.9 R e^{-1},} \\
W=0.118 R e .
\end{gathered}
$$

The lengthscale here is the cylinder radius and $c_{d}=\mathrm{drag} /\left(\rho U_{\mathrm{o}}^{2} R\right)$. The slowing down of the flow on the body scale under the influence of the eddy is one of the main features of this theory. However, recalling that $[B]$ is the jump in the Bernoulli constant on the body scale at $s=n=0$, it follows from (5.4) that at $R e=150$ the velocity on the free streamline on the body scale equals 1 (through asymptotically it is small). For this reason good agreement with numerical calculations is likely only for $R e$ considerably 


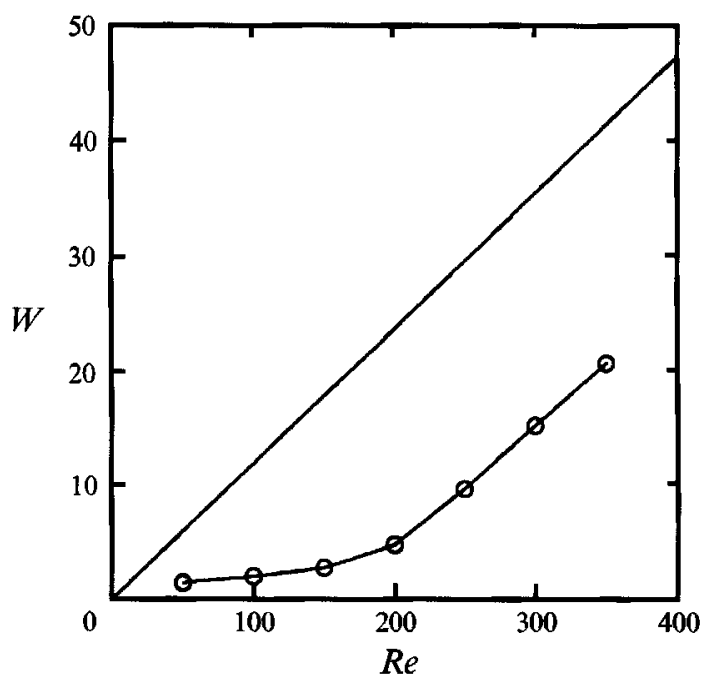

Figure 5. The eddy half-width in the flow past an isolated body. The line is (5.5), points are Fornberg's (1991) results. Only the ultimate slope is to be compared.

greater than 150 , so it cannot really be expected for the Reynolds numbers achieved so far. It also should be pointed out that the theory gives only the ultimate slope of the curves $L(R e)$ and $W(R e)$.

From the renormalized Smith's (1979) results (see \$2.3) it follows that the separation point is at a distance of order $R e^{-\frac{1}{32}}$ downstream from its limiting $(R e=\infty)$ position. Accordingly, for moderate $R e$ the proper value of $k_{d}$ depends weakly on $R e$ and is somewhat less than its limiting value for $R e=\infty$. Considering the flow past a circular cylinder, Smith (1979) made all the necessary calculations for the improvement which can be made by using a more appropriate value for $k_{d}$. Unfortunately, he presented them only summed with the $O\left(R e^{-\frac{1}{2}}\right)$ corrections specific to his theory. For this reason, apart from the limiting value $k_{d}=0.50$, quantitative results for $k_{d}$ are not available. Instead, the value $k_{d}=0.45$ was chosen so as to fit better the ultimate slope of $L v$ s. $R e$ for the flow past an isolated circular cylinder and has been used for all comparisons with Fornberg's data in the next section. This assumes that $k_{d}$ varies little in the range of Reynolds numbers considered but, in any case, the change in $k_{d}$ only slightly affects our quantitative results.

The variation of $L$ and $W$ with $R e$ is given in figures 4 and 5 , in which the solid lines without points are the theory for $k_{d}=0.5$, the dashed line (in figure 4 ) is the theory for $k_{d}=0.45$ and the points are the numerical calculations of Fornberg $(1985,1991)$. Comparisons for other quantities are given in the next section. Note the transition in the behaviour of the numerical results at $R e=150$, displayed most clearly in figure 5 . This transition will be discussed in the following section.

According to our theory the Bernoulli constant is continuous in the rear turn region. For this reason in the rear part of the eddy boundary there is no cusp and this feature can easily be seen in the numerical results.

\subsection{Further comparisons and discussion}

Quantitative results for the cascade flow are based on Sadovskii flow calculations in a channel (Chernyshenko 1993). The values of $D_{0}(b)$ were obtained by interpolating the data of Chernyshenko (1982) (see also the Appendix). 


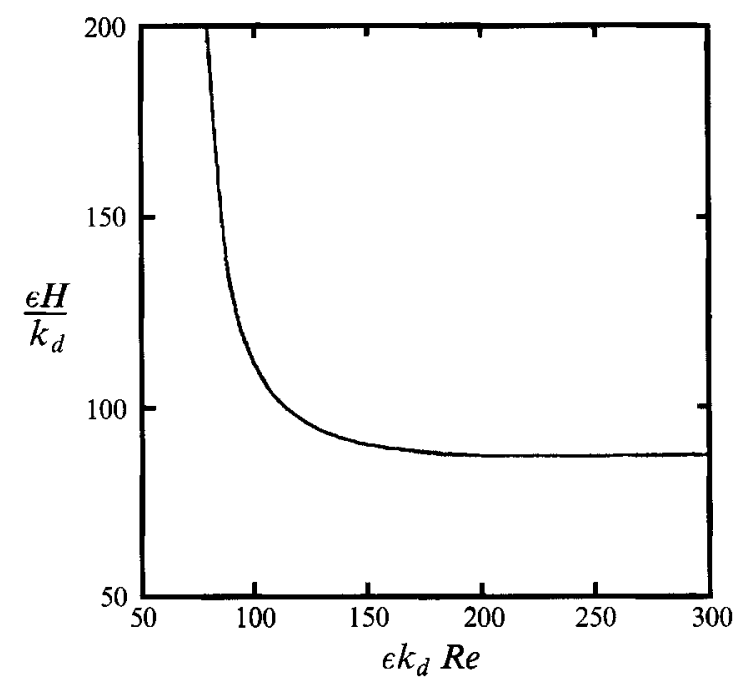

Figure 6. Region of validity of equation (5.6). The theory is not satisfied below this curve.

For the comparison of the asymptotic results with numerical calculations the vital question is: how large must $R e$ and $H$ be before reasonable agreement can be expected? It seems, quite unusually, that the answer can be found in the leading term of the expansion. According to the theory the velocity on the free streamline near the body equals $(-2[B])^{\frac{1}{2}}=\left[C /\left(k_{d} R e\right)\right]^{\frac{1}{2}}$ and therefore tends to zero. Hence good agreement can be expected only for

with $\epsilon$ sufficiently small.

$$
C /\left(k_{d} R e\right)<\epsilon
$$

For the flow past an isolated body $C \approx 73$ (Chernyshenko's 1993 data are used throughout this section, even for $H / L=\infty$-Sadovskii's value of $C=74.9$ is not too different in any case). The comparison with the numerical calculations (see figures 4 and 5) shows a satisfactory agreement only for the eddy length. For other quantities the numerical results display a tendency to approach the asymptotics only for $R e>150$ (in our notation). This exactly corresponds to $C /\left(k_{d} R e\right)<1$. Good quantitative agreement is not achieved even for the highest calculated Re. Multiplying (2.22) by $\epsilon k_{d} R e$ yields $\epsilon H / k_{d}=H /\left[2 L D_{0}^{2}(b)(\alpha C)^{\frac{1}{2}}\right] \epsilon k_{d} R e$. Together with $C /\left(k_{d} R e\right)=\epsilon$ this gives the dependence of $\epsilon H / k_{d}$ on $\epsilon k_{d} R e$ (recall that $\alpha, b$ and $C$ depend only on $H / L$ ), which can be obtained by varying $H / L$ from 0 to $+\infty$ and is shown in figure 6 . Above the curve in figure 6 expression (5.6) is satisfied. This curve has two asymptotes: for $\epsilon H / k_{d} \rightarrow \infty, \epsilon k_{d} R e \approx 73$ and for $\epsilon k_{d} R e \rightarrow \infty, \epsilon H / k_{d} \rightarrow 9 /\left[2 D_{0}^{2}(0.5)\right]=91.3$. If we assume by analogy with the flow past an isolated body that the comparison must be made at least for $C /\left(k_{d} R e\right)<1$, then practically all Fornberg's (1991) calculations and all the calculations of Natarajan et al. (1992) lie outside the range of validity of this theory. The reason is that with $H / R e$ (and, correspondingly, $H / L$ ) decreasing $C$ increases (see the asymptotics for $C$ preceding equation (3.4)).

On the other hand, the influence of the neighbouring bodies depends mostly on the ratio $W / H$. For moderate Reynolds numbers the theory overestimates $W$ (see figure 5) so we may hope that the proper value of $C$ in (5.6) may be calculated in terms of the numerically calculated $W / H$ rather than the value of $H /\left(k_{d}^{2} R e\right)$. In other words, instead of using the member of the family of Sadovskii flows determined by $H / L$ from (2.22), the member with the same $W / H$ as that obtained in the full Navier-Stokes calculation may be used to estimate $C$ in (5.6). Figure 7 shows a comparison between 


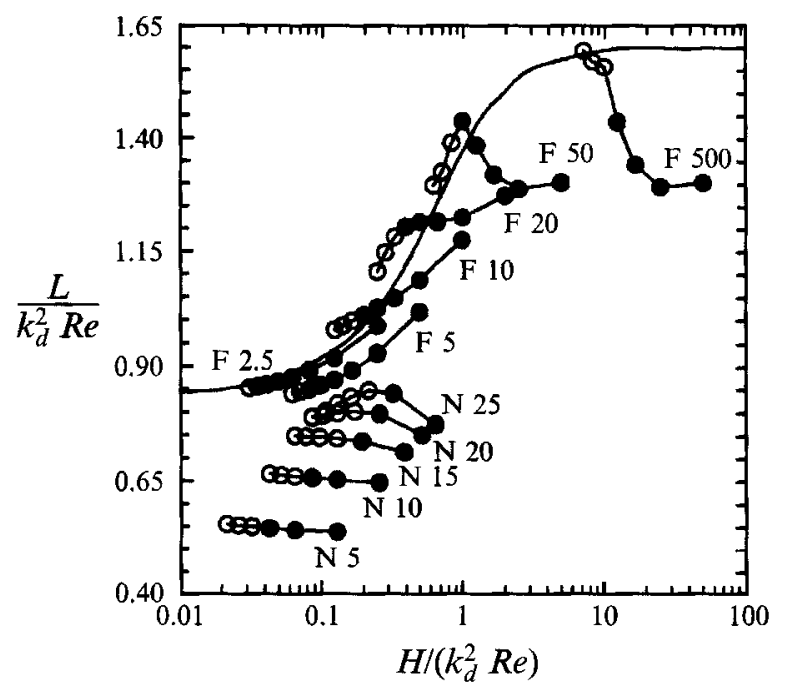

FIGURE 7. The eddy length as a function of $H /\left(k_{d}^{2} R e\right)$. Natarajan et al.'s (1992) results are marked with $\mathrm{N}$ and those of Fornberg (1991) with $\mathrm{F}$. The numbers near these letters are the values of $H$. The curve is given by (2.21) and (2.22) with $H / L$ varying from 0 to 1 . For the open symbols $C\left(W_{\text {cale }} / H_{\text {calc }} /\left(k_{d} R e\right)<0.72\right.$. For Natarajan et al.'s results $R e=50-300$ at intervals of 50 , from filled to open symbols. For Fornberg's results the values of $R e$ are the same as in figure 8.

the asymptotic predictions for the eddy length and the numerical results of Fornberg (1991) and Natarajan et al. (1992). Fornberg's data for $H=\infty$ are not included because they are identical with the data for $H=500$. (Note the difference between our notation and that of Fornberg: $R e_{F}=2 R e, W_{F}=2 H$, where the subscript refers to Fornberg's variables.) For the open symbols $C\left(W_{C} / H_{C}\right) /\left(k_{d} R e\right)<0.72$, where the subscript $C$ denotes the calculated values. For the solid symbols this condition is not satisfied. For each set of fixed $H$ in figure 7 the Reynolds number increases from solid to open symbols. For large $H$ the open symbols clearly display a tendency toward the asymptotic results with $R e$ and $H$ increasing, hence supporting the theory (the calculated values of $W / H$ were used only to decide whether the symbol must be solid or open, not to determine the symbol location). The values of $H$ in Natarajan $e t$ al.'s (1992) calculations (for a flat plate) were not large enough to obtain agreement. Evidently, a flat plate creates a bigger effective blockage than a circular cylinder of the same size - not surprisingly, given the initially much wider wake resulting from separated streamlines starting normal to the oncoming flow. Good quantitative agreement can be obtained only for much larger $R e$ (and $H$ in Natarajan $e t$ al.'s 1992 calculations) than was achieved in the numerical calculations. Nevertheless, Natarajan et al.'s (1992) results clearly show some tendency towards the theoretical predictions with $H$ increasing. Concerning the applicability of the condition (5.6), the results are far less conclusive. Indeed, (5.6) implies by an analogy with the flow past an isolated body, that numerical results must experience a transition in behaviour between solid and open symbols. The sets of data for fixed $H$, displaying the transition, display it at values of $R e$ in accordance with (5.6), with $\epsilon$ close to 1 . On the other hand, the sets with small $H$ do not display the transition at all, although it is predicted by (5.6).

For further analysis let us consider the behaviour of the solution for fixed $H$ and increasing $R e$ implied by our theory. For sufficiently large $H$ but small $R e$ the condition (5.6) is not satisfied and the solution may be far from the asymptotic theory. For larger $R e$ the condition (5.6) is satisfied and we can expect transition to the asymptotic 


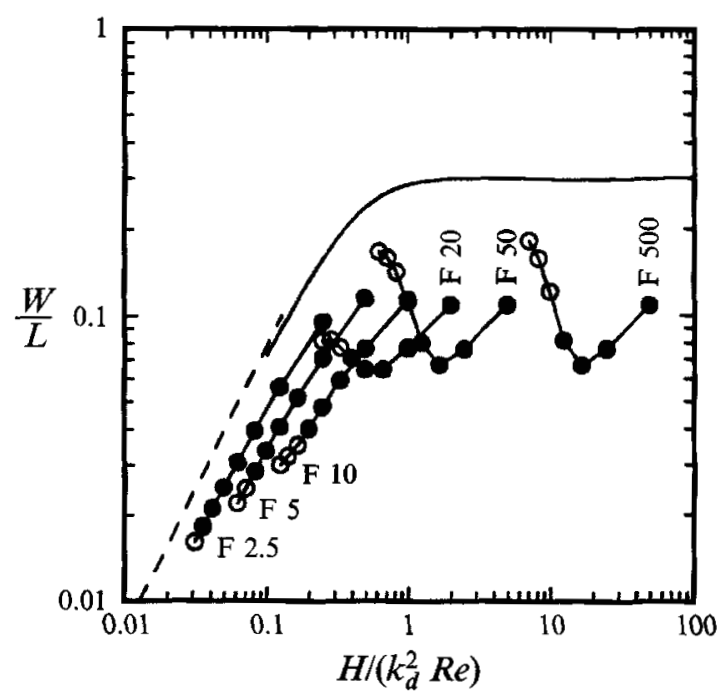

FIGURE 8. The ratio of eddy half-width to the eddy length as a function of $H /\left(k_{d}^{2} R e\right)$. The notation is the same as in figure 7. The solid curve is derived from (2.22) combined with the dependence of $W / L$ on $H / L$ in the Sadovskii flow calculated by Chernyshenko (1993). The dashed line is derived from the asymptotics for $H / R e \rightarrow 0$ given in $\$ 3$. Symbols are Fornberg's (1991) data: $R e=50-400$ at intervals of 50 , from filled to open symbols; for $H=500, R e=50-350$.

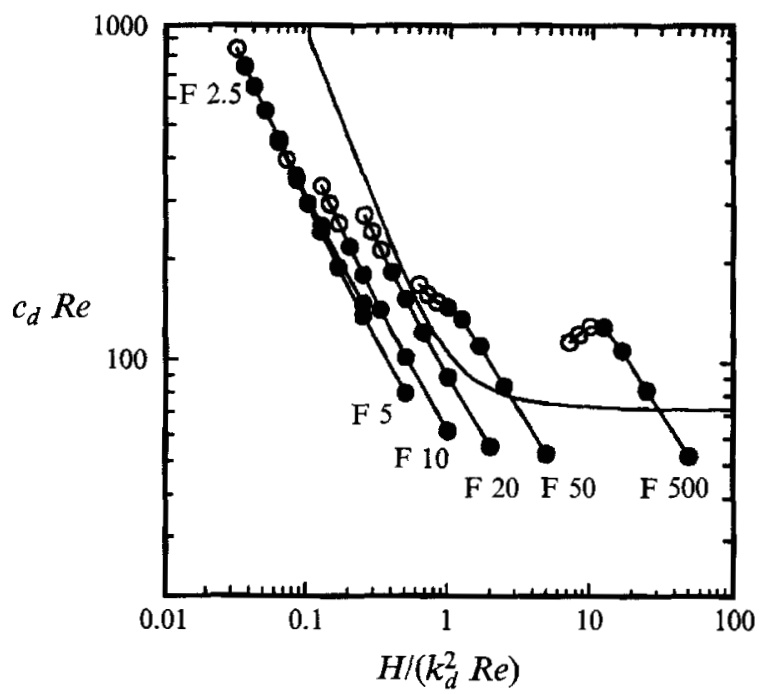

FIGURE 9. $c_{d} R e$ as a function of $H /\left(k_{d}^{2} R e\right)$. The curve is given by (2.16) and (2.22). $\operatorname{Re}=50-400$ at intervals of 50, from filled to open symbols. Symbols are Fornberg's (1991) data: for $H=500$, $R e=50-350$.

behaviour. The eventual accuracy depends on the particular value of $H$ because for very small $H / R e$ the accuracy of our theory depends on $H$ rather than on $R e$, as can be seen from the results of $\S 3$. For $H$ less than some value, equation (5.6) is not satisfied for any $R e$, see figure 6. Hence for these smaller values of $H$ there is no reason to expect the change in the behaviour of the solution with $R e$ increasing. These qualitative predictions are in good agreement with the numerical results. From figure 8 , in which the ratio of eddy half-width to eddy length is compared, we can see that the results for 


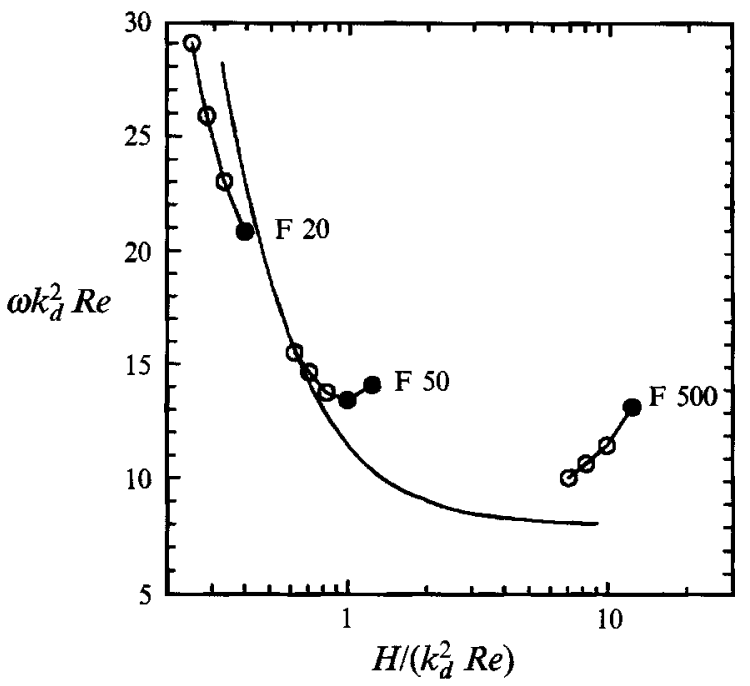

FIGURE 10. $\omega k_{d}^{2} R e$ as a function of $H /\left(k_{d}^{2} R e\right)$. The curve is given by (2.18) and (2.22). Symbols are Fornberg's (1991) data: for $H=20, R e=250-400 ; H=50, R e=200-400 ; H=500, R e=200-350$; at intervals of 50 from filled to open symbols.

$H=500,50$ and 20 do indeed experience a transition in behaviour whilst the results for $H=10,5$ and 2.5 do not. Correspondingly, when $H$ changes for sufficiently large but constant $R e$, the transition occurs in the region $10<H<20$, or $2.3<C /\left(k_{d} R e\right)<4.6$ (the $H \ll R e$ formulae from $\S 3$ are used here). This may be compared with the transition in the flow past an isolated body, which occurs for $0.75<C /\left(k_{d} R e\right)<1$, as seen from Fornberg's results combined with $C \sim 73$. Taking into account the uncertainty of the region of transition the difference is not unreasonably large. Nevertheless, the nature of the observed transition in the numerical results, when $H$ changes and $R e$ is large and fixed, may be (and in all probability is) quite different, as discussed below. If so, the transition in the solution with $H$ increasing, predicted by (5.6), appears only at very high $R e$. Natarajan $e t$ al.'s (1992) results are not shown in figure 8, nor in the following figure 9, because they overlap with those of Fornberg (1991) for smaller $H$ and have a similar behaviour. In figure 9 the asymptotic results for $c_{d}$ are shown in comparison with the numerical ones and in figure 10 results for the vorticity in the eddy are compared with those calculated by Fornberg (1991, private communication). The number of computed points is less than in the previous figures because in many cases Fornberg's calculations did not exhibit a plateau in the vorticity distribution.

The restriction (5.6) is due to the velocity on the body scale being asymptotically small. This is not so in the asymptotic theory for $H \sim 1, R e \rightarrow \infty$, outlined in $\$ 4$. Hence the $H \sim 1, R e \rightarrow \infty$ asymptotics may give good quantitative results for smaller $R e$ than the theory for the flow past an isolated body. For this reason it is interesting to analyse the previous attempts to solve this problem. Milos \& Acrivos (1986) proposed an asymptotic structure for the flow in a sudden expansion very similar to that described in $\S 4$ above. It should be noted however that their assumption that the initial eddy width on the eddy scale, a parameter in their calculations, equals the step height, is not correct. The flow on the eddy scale matches the flow on the step scale. The inviscid nature of the step-scale flow provides an initial condition for the eddy-scale flow and the condition is that the velocity on the streamlines emerging from the step-scale region 
into the eddy equals the velocity on the same streamline entering the step-scale region, as was correctly assumed by Milos \& Acrivos (1986). At the same time the eddy width on the step scale increases from the step height to its downstream value owing to the acceleration of the vortex flow inside the eddy. Assuming that the velocity profile is uniform immediately above the step, which is valid only approximately because of the upstream influence in the step-scale flow, it is quite easy to recalculate the step height corresponding to the initial eddy width in Milos \& Acrivos' calculations, using Bernoulli's equation and mass conservation. Hence the Milos \& Acrivos results are nevertheless meaningful and, in fact, very important. They show that, provided the blockage ratio is sufficiently large, the solution of the boundary-layer problem arising in the theory does exist even if the sudden-inviscid-reattachment downstream boundary condition, implied by our theory, is not imposed. Therefore it is possible that with $H$ decreasing the inviscid reattachment region disappears. Such a possibility is supported by the good agreement between the asymptotic theory and the numerical calculations of Milos \& Acrivos (1986) and Milos et al. (1987) and also Ingham et al. (1990). Ingham et al. compared their numerical results with the predictions given by Smith $(1985 a)$. In Smith's asymptotic theory the reattachment also was not inviscid. He assumed the flow on the body scale to be of the Kirchhoff type, but this may be sufficiently accurate for the case considered by Ingham et al. If indeed for small $H$ there is no inviscid reattachment region, then the transition observed in the numerical results for decreasing $H$ may be associated with the disappearance of this region rather than with (5.6). Another result of Smith is also in favour of this possibility. If the asymptotic structure changes with $H$ increasing, then the solution must develop a singularity when $H$ approaches some critical value. Smith (1988) showed that appearance of a singularity is possible in the solutions of similar equations.

Summing up, we may say that the transition for fixed $H$ and changing $R e$ may be attributed to (5.6) and explained as a transition from a flow in which the distributed energy dissipation, characterized by the constant $C$, prevents rapid expansion of the eddy with $R e$ increasing, to a flow in which this effect is insignificant.

The transition for fixed $R e$ and changing $H$ has two possible explanations. The first one is the same as that just given. The second is that this transition is from a flow without a sudden reattachment region to a flow with one. The latter seems to be more likely for the transition observed so far in numerical calculations. The former may nevertheless appear as a second transition at larger but finite $H$ and become distinguishable from the latter only at very large $R e$. Further investigation is needed to explain this phenomenon completely.

It remains to consider the implications of our results for the entire problem of separated flows. First our results indicate that although the Navier-Stokes equations do not include explicitly large constants or coefficients apart from the Reynolds number, which is a parameter, at least one large constant is present implicitly. For the flow past an isolated body $C \approx 73$. From the physical point of view $C$ is the energy dissipation coefficient in the entire flow field outside the thin regions of high velocity gradients. The usual estimate for the rate of energy dissipation in such regions is $1 / R e$. Usually this is small, but our theory shows that in separated flows it must be multiplied by $C$. Therefore the energy dissipation outside the shear layers is very significant: until the condition $R e \gg C$ is satisfied the distributed energy dissipation prevents the rapid expansion of the eddy. This means that numerical schemes must have sufficient resolution to achieve implied accuracy in the energy dissipation throughout the entire flow field, if these schemes are to be used for calculations at $\operatorname{Re} \sim C$. The distributed energy dissipation may also be essential in turbulent separated flows, for which the 
effective Reynolds numbers are usually comparable with $C$, although in this case the important region is restricted to the interior of the eddy due to the very small turbulent viscosity outside. However, great caution is needed here, because the analogy between laminar and turbulent separated flows is unreliable. The requirements for accuracy on the body scale are not so strict; the correct determination of the separation point must be provided and the inviscid terms in the Navier-Stokes equation must be approximated accurately.

For flows in narrow channels and cascades only the terms present in the boundarylayer equations must be approximated properly over most of the flow. Near the body the inviscid terms are important and the correct position of the separation point is essential. Near the reattachment point an accurate modelling of the inviscid terms is essential. The energy dissipation in channel flows is correctly described by boundarylayer equations. Naturally, in the full Navier-Stokes computations no terms can be completely neglected. Our results indicate only the comparative importance of the different terms.

\subsection{A final methodological note}

To calculate the ratio of the eddy length to the body size it is insufficient to match the leading terms of the expansion in the body-scale flow, the intermediate region and the cusp region, because the intermediate region can be constructed for any ratio provided that the size of the cusp region is asymptotically large. In the theory presented in this paper, this ratio was determined by using the condition of the equality of the drag coefficients calculated via the flow on the body scale and via the far wake. This condition does not follow from matching the leading terms, so if only the leading terms are considered it is possible to construct a model with one arbitrary parameter whose correct, unique value could then be found by the usual procedure of analysing the next terms of the expansion. Such a procedure would not be easy and to avoid this difficulty it is sometimes useful to formulate a relation which is valid for the exact solution and to demand it to be valid in the limit. For example, the well-known Prandtl-Batchelor theorem is usually proved by this method, and the same idea was used here to deduce (2.19). To understand why the equality of drags calculated on the body scale and via the far wake is necessary for the self-consistency of higher terms of the expansion, let us consider the momentum flux through the contour enclosing the body. The main terms of the expansion on the body scale and in the far wake yield the same order $R e^{-1}$ of the momentum flux, which equals the drag, while the main term for the momentum flux on the eddy scale is of order $R e$ but has zero value. It is now clear that one of the higher terms on this scale must yield the momentum flux or order $R e^{-1}$ and it is impossible to match this term with expansions on other scales if the condition of equality of drags calculated via different regions is not satisfied.

The bulk of this work was undertaken during a visit by the first author to the University of Surrey, supported by the Royal Society of London. We are grateful for this support and S.I.C. also thanks the whole staff of the Department of Mechanical Engineering of the University of Surrey for their hospitality during his stay. The authors also wish to thank the referees for their many helpful suggestions during the review process. 


\section{Appendix. The relationship between the vorticity in the eddy and other} eddy properties

Let us consider a somewhat more general problem than (2.6)-(2.11):

$$
\begin{gathered}
\frac{\partial \omega}{\partial s}=\frac{U(s)}{\operatorname{Re}} \frac{\partial^{2} \omega}{\partial \psi^{2}}, \\
s=0, \quad \psi<0, \quad \omega=\Omega(\psi), \\
\psi>0, \quad \omega(0, \psi)=\omega\left(s_{A}, \psi\right), \\
\psi=0, \quad s_{B}<s<s_{A}, \quad \omega(s, 0)=0, \\
\psi \rightarrow \infty, \quad \omega \rightarrow \omega_{\infty} .
\end{gathered}
$$

Here the condition (2.8) is changed to the more general condition (A 2). The unknown function $\omega(s, \psi)$ is defined in the domain $\Omega_{1} \cup \Omega_{2}$ :

$$
\Omega_{1}=\left\{s, \psi: 0 \leqslant s \leqslant s_{B},-\infty<\psi<+\infty\right\}, \quad \Omega_{2}=\left\{s, \psi: s_{B} \leqslant s \leqslant s_{A}, 0 \leqslant \psi<+\infty\right\} .
$$

The unknown value of $\omega_{\infty}$ is to be found as a functional of $\Omega(\psi), U(s)$ and a function of $s_{A}, s_{B}$. Let us introduce the new variables $\tau, p$ :

$$
\tau=t / t_{A}, \quad t=\int_{0}^{s} U(s) \mathrm{d} s, \quad \psi=2 p t_{A}^{\frac{1}{2}}, \quad t_{A}=t\left(s_{A}\right) .
$$

Then (A 1) and the boundary conditions (A 2)-(A 5 ) take the form

$$
\left.\begin{array}{rl}
\frac{\partial \omega}{\partial \tau} & =\frac{1}{4} \frac{\partial^{2} \omega}{\partial p^{2}}, \\
\omega(0, p) & =h(p)=\Omega\left(\frac{p}{2 t_{A}^{\frac{1}{2}}}\right), \quad p \leqslant 0 ; \quad \omega(0, p)=\omega(1, p), \quad p>0, \\
\omega(\tau, 0) & =0, \quad b<\tau<1, \quad b=t_{B} / t_{A} .
\end{array}\right\}
$$

Denote $\omega(0, p)=y(p)$ and $\omega(b, p)=z(p)$. Using the fundamental solution of the heat equation (Tihonov \& Samarskii 1972), we can express $z(p)$ via $y(p)$ and $h(p)$ and, using Green's function for the half-infinite interval (Tihonov \& Samarskii 1972), we can express $\omega(1, p)$ via $z(p)$. From (A 7$)$ it follows that $y(p)=\omega(1, p)$. Changing the order of integration in the double integral, we arrive at the integral equation

$$
\begin{aligned}
y(p) & =\int_{0}^{\infty} R(p, \xi) y(\xi) \mathrm{d} \xi+f(p), \\
R(p, \xi) & =\frac{1}{2 \pi^{\frac{1}{2}}}\left[\mathrm{e}^{-(p-\xi)^{2}} \operatorname{erfc}\left(\frac{-b p-(1-b) \xi}{[b(1-b)]^{\frac{1}{2}}}\right)-\mathrm{e}^{-(p+\xi)^{2}} \operatorname{erfc}\left(\frac{b p-(1-b) \xi}{[b(1-b)]^{\frac{1}{2}}}\right)\right], \\
\operatorname{erfc}(t) & =\int_{0}^{t} \frac{2 \mathrm{e}^{-x^{2}}}{\pi^{\frac{1}{2}}} \mathrm{~d} x, \quad f(p)=\int_{-\infty}^{0} R(p, \xi) h(\xi) \mathrm{d} \xi .
\end{aligned}
$$

Consider the solution $G(p)$ of the integral equation with a transposed kernel

$$
G(p)=\int_{0}^{\infty} R(\xi, p) G(\xi) \mathrm{d} \xi
$$

such that $G(p) \rightarrow p$ for $p \rightarrow+\infty$. Attempts to prove the existence of such a solution were 
not successful. The existence of the solution is substantiated to some extent by the fact that it was obtained numerically, and the results therewith did not lead to contradictions. The uniqueness of the solution is of no consequence. If there are several solutions, any one of them may be used.

Let

$$
\begin{aligned}
& y(p)=y_{\infty}+y_{1}(p), \quad y_{\infty}=\lim _{p \rightarrow \infty} y(p), \quad R(p, \xi)=R_{\infty}(p, \xi)+R_{1}(p, \xi), \\
& R_{1} \rightarrow 0, \quad p, x \rightarrow \infty, \quad R_{\infty}(p, \xi)=\frac{1}{\pi^{\frac{1}{2}}} \mathrm{e}^{-(p-\xi)^{2}} .
\end{aligned}
$$

Substituting $f(p)$ from (A 8$)$ and $G(p)$ from (A 9) we deduce that

$$
J=\int_{0}^{\infty} G(p) f(p) \mathrm{d} p=\int_{0}^{\infty} \int_{0}^{\infty}[y(p) G(\xi) R(\xi, p)-y(\xi) G(p) R(p, \xi)] \mathrm{d} \xi \mathrm{d} p .
$$

If each term of the difference were integrated separately, the double integrals would be equal and would cancel each other. However, these integrals diverge. Let us express $R$ as a sum of $R_{\infty}$ and $R_{1}$. The integrals of the terms with $R_{1}$ converge and therefore cancel each other. Analogously, separating out $y_{\infty}$ from $y$, we arrive at

$$
J=y_{\infty} \int_{0}^{\infty} \int_{0}^{\infty}[G(\xi)-G(p)] R_{\infty}(p, \xi) \mathrm{d} \xi \mathrm{d} p=\text { const } \times y_{\infty},
$$

As $y_{1}(p) \rightarrow 0$ for $p \rightarrow \infty$ exponentially, the corresponding integrals converge and hence cancel each other. This is easy to prove taking into account that $y(p)=\omega(0, p)$, and that $\omega(\tau, p)$ behaves as a solution of the problem without initial conditions for the heat equation in a half-infinite interval (Tihonov \& Samarskii 1972). Assuming that the function $G_{1}(p)-\lim _{x \rightarrow \infty} G_{1}(x)$ may be integrated from 0 to $\infty$, the method of separating out the main part may be repeated. As a result we obtain const $=0.25$. The numerical calculations support this assumption.

Therefore

$$
y_{\infty}=4 \int_{0}^{\infty} G(p) f(p) \mathrm{d} p .
$$

Since $y_{\infty}=\omega_{\infty}$, we obtain the following formula:

$$
\omega_{\infty}=4 \int_{0}^{\infty} G(p)\left\{\int_{-\infty}^{0} R(p, \xi) h(\xi) \mathrm{d} \xi\right\} \mathrm{d} p .
$$

If the order of integration can be changed, (A 11) can be simplified:

$$
\omega_{\infty}=4 \int_{\infty}^{0} D(\xi) h(\xi) \mathrm{d} \xi
$$

Equation (A 9) was solved numerically by the method of successive approximations. Equation (A 10) permits one to verify indirectly the calculations. Let us take an arbitrary function $y(p) \rightarrow$ const for $p \rightarrow \infty$ and determine the corresponding function $f(p)$ from (A 8). The value of $y_{\infty}$ calculated from (A 10) must coincide with the value of $y(p)$ at infinity. In the numerical calculations the discrepancy was less than $1 \%$. As in the original problem the function $\Omega(\psi)$ is a delta-function, only the value of $D(0)$ is of importance. This was denoted $D_{0}(b)$ throughout this paper, and the calculated 


\begin{tabular}{lllllll}
\hline$b$ & 0.1 & 0.2 & 0.3 & 0.5 & 0.7 & 0.9 \\
$D_{0}(b)$ & 0.0090 & 0.1294 & 0.1619 & 0.2217 & 0.2857 & 0.3744 \\
& & \multicolumn{7}{c}{ TABLE 1} \\
\hline
\end{tabular}

values are given in table 1 . Turning back to the original problem, by simple substitution we easily arrive at (2.12).

In Chernyshenko (1982) the problem was addressed in more general form allowing the boundary values of $\omega$ at $\psi=0, s_{b}<s<s_{A}$ to be non-zero and the results were represented more completely. Two misprints in Chernyshenko (1982) should be noted. The formula of the substitution of the variable for $p$ should be as in this appendix, and in table 2 the values of $E(\tau)$ and not of $100 E(\tau)$ are given.

\section{REFERENCES}

ACrivos, A., Leal, L. G., Snowden, D. D. \& PAN, F. 1968 Further experiments on steady separated flow past bluff objects. J. Fluid Mech. 34, 25.

Acrivos, A., Snowden, D. D., Grove, A. S. \& Petersen, E. E. 1965 The steady separated flow past a circular cylinder at large Reynolds numbers. J. Fluid Mech. 21, 737-760.

BATCHELOR, G. K. 1956 A proposal concerning laminar wakes behind bluff bodies at large Reynolds number. J. Fluid Mech. 1, 338-398.

Bukovshin, V. G. \& Taganov, G. I. 1976 Numerical results of the asymptotic theory of the flow past a body with stationary separation eddy at large Reynolds number. Chislennye metody mechaniki sploshnoi sredy. Novosibirsk: VC SO AN SSSR 7, 13-26 (in Russian).

CHERNYSHENKo, S. I. 1982 An approximate method of determining the vorticity in the separation region as the viscosity tends to zero. Fluid Dyn. 17 (1), 7-11. (Izv. Akad. Nauk SSSR, Mekh. Zhidk. Gaza 1, 10-15.)

Chernyshenko, S. I. 1984 Calculation of low-viscosity flows with separation by means of Batchelor's model. Fluid Dyn. 19 (2), 206-210. (Izv. Akad. Nauk SSSR, Mekh. Zhidk. Gaza 2, 40-45.)

CHERnyshenko, S. I. 1988 The asymptotic form of the stationary separated circumfluence of a body at high Reynolds number. Appl. Math. Mech. 52, 746. (Prikl. Matem. Mekh. 52 (6), 958-966.)

CHERnyshenKo, S. I. 1991 Separated flow over a backward-facing step whose height is much greater than the thickness of the lower sublayer of the interaction zone. Fluid Dyn. 26 (4), 496-501. (Izv. Akad. Nauk SSSR, Mekh. Zhidk. Gaza 4, 25-30.)

Chernyshenko, S. I. 1993 Stratified Sadovskii flow in a channel. J. Fluid Mech. 250, 423-431.

FORNBERG, B. 1985 Steady viscous flow past a circular cylinder up to Reynolds number 600 . J. Comput. Phys. 61, 297-320.

FORNBERG, B. 1991 Steady incompressible flow past a row of circular cylinders. J. Fluid Mech. 225, 655-671.

Fornberg, B. 1993 Computing steady incompressible flows past blunt bodies - a historical overview. In Numerical Methods for Fluid Dynamics IV (ed. M. J. Baines \& K. W. Morton), pp. 115-134, Oxford University Press.

HeRwiG, H. 1982 Die Anwendung der asymptotischen Theorie auf laminare Stromungen mit endlichen Ablosegebieter. Z.Flugwiss. Weltraumforsh. B 46, (4), 266-279 (in German).

INGHAM, D. B., TANG, T. \& MoRTon, B. R. 1990 Steady two-dimensional flow through a row of normal flat plates. J. Fluid Mech. 210, 281-302.

Kolosov, B. V. \& Shifrin, E. G. 1975 On a particular boundary value problem arising in the investigation of closed stationary separation zones in an incompressible fluid. Appl. Math. Mech. 39, (5), 802-811. (Prikl. Mat. Mech. 39 (5), 773-779.)

LUKerChenko, N. N. 1990 A comparison of two structures for impinging jets with different Bernoulli's constants. Zh. Prikl. Mech. i Techn. Phys. No. 6, 97-101 (In Russian). 
Milos, F. S. \& ACrivos, A. 1986 Steady flow past sudden expansions at large Reynolds numbers. Part I. Boundary layer solutions. Phys. Fluids 29, 1353-1359.

Milos, F. S., ACrivos, A. \& KIM, J. 1987 Steady flow past sudden expansions at large Reynolds numbers. Part II. Navier-Stokes solutions for the cascade expansion. Phys. Fluids 30, 7-18.

MoOre, D. W., SAFfman, P. G. \& TANVEER, S. 1988 The calculation of some Batchelor flows: The Sadovskii vortex and rotational corner flow. Phys. Fluids 31, 978.

Natarajan, R., Fornberg, B. \& Acrivos A. 1992 Flow past a row of flat plates at large Reynolds number. Proc. $R$. Soc. Lond. A 441, 211-235.

Peregrine, D. H. 1985 A note on the steady high-Reynolds-number flow about a circular cylinder. J. Fluid Mech. 157, 493-500.

SADOVSKII, V. S. 1970 The region of constant vorticity in plain potential flow. Uch. Zap. TsAGI 1 (4), 1-9 (in Russian).

SAdovsKII, V.S. 1971 a Vortex regions in a potential stream with a jump in Bernoulli's constant across the boundary. Appl Math. Mech. 35, 729. (Prikl. Matem. Mekh. 35 (5), 773-779.)

SADOVSKII, V.S. $1971 b$ Some properties of vortex and potential flows touching along a closed fiuid streamline. Uch. Zap. TsAGI 2 (1), 113-116 (in Russian).

SADOVSKII, V.S. 1973 A study of the solutions to Euler's equations containing regions of constant vorticity. Trudy TSAGI. 1474, 1-14 (in Russian).

SADovsKin, V. S. \& KozhURo, L. A. 1977 On two one-parameter families of vortex flows of inviscid fluid. Chislennye metody mechaniki sploshnoi sredy, Novosibirsk. 8 (7). 126-140 (in Russian).

SAFFMan, P. G. \& TANVEER, S. 1982 The touching pair of equal and opposite uniform vortices. Phys. Fluids 25, 1929.

SERrin, J. 1959 Mathematical Principles of Classical Fluid Mechanics.

SMITH, F. T. 1979 Laminar flow of an incompressible fluid past a bluff body: the separation, reattachment, eddy properties and drag. J. Fluid Mech. 92, 171-205.

Sмith, F. T. 1985a On large-scale eddy closure. J. Math. Phys. Sci. 19, 1-80.

Sмiтн, F. T. $1985 b$ A structure for laminar flow past a bluff body at high Reynolds number. J. Fluid Mech. 155, 175-191.

SMITH, F. T. 1988 A reversed flow singularity in interacting boundary layers. Proc. R. Soc. Lond. A 420, 21-52.

Sychev, V. V. 1967 Rep. to 8th Symp. Recent Problems in Mech. Liquids \& Gases, Tarda, Poland.

Taganov, G. I. 1968 Contribution to the theory of stationary separation zones. Fluid Dyn. 3, (5), 1-11 (Izv. AN SSSR. Mekhanika Zhidkosti i Gaza 3 (5), 3-19).

TAGANOV, G. I. 1970 On limiting flows of viscous fluid with stationary separation for $R e \rightarrow \infty$. Uch. Zap. TsAGI. 1 (3), 1-14 (in Russian).

Tinonov, A. N. \& SAmarskil, A. A. 1972 Equations of Mathematical Physics. Moscow; Nauka (in Russian).

TuRfus, C. 1993 Prandtl-Batchelor flow past a flat plat at normal incidence in a channel - inviscid analysis. J. Fluid Mech. 249, 59-72. 